\title{
EXTENDED DIVERGENCE-MEASURE FIELDS AND THE EULER EQUATIONS FOR GAS DYNAMICS
}

\author{
GUI-QIANG CHEN \& HERMANO FRID
}

\begin{abstract}
A class of extended vector fields, called extended divergence-measure fields, is analyzed. These fields include vector fields in $L^{p}$ and vector-valued Radon measures, whose divergences are Radon measures. Such extended vector fields arise naturally in the study of the behavior of entropy solutions to the Euler equations for gas dynamics and other nonlinear systems of conservation laws. A new notion of normal traces over Lipschitz deformable surfaces is developed under which a generalized Gauss-Green theorem is established even for these extended fields. An explicit formula is obtained to calculate the normal traces over any Lipschitz deformable surface, suitable for applications, by using the neighborhood information of the fields near the surface and the level set function of the Lipschitz deformation surfaces. As an application, we prove the uniqueness and stability of Riemann solutions that may contain vacuum in the class of entropy solutions of the Euler equations for gas dynamics.
\end{abstract}

\section{INTRODUCTION}

We are concerned with a class of extended vector fields, called extended divergencemeasure fields, or $\mathcal{D} \mathcal{M}$-fields for short. These fields include vector fields in $L^{p}, 1 \leq$ $p \leq \infty$, and vector-valued Radon measures, whose divergences are Radon measures. The $\mathcal{D} \mathcal{M}$-fields arise naturally in the study of the behavior of entropy solutions of nonlinear hyperbolic systems of conservation laws, which take the form

$$
u_{t}+\nabla_{x} \cdot f(u)=0, \quad u \in \mathbb{R}^{m}, x \in \mathbb{R}^{n},
$$

where $f: \mathbb{R}^{m} \rightarrow\left(\mathbb{R}^{m}\right)^{n}$ is a nonlinear map. One of its most important prototypes is the Euler equations for gas dynamics in Lagrangian coordinates:

$$
\begin{aligned}
& \tau_{t}-v_{x}=0, \\
& v_{t}+p_{x}=0, \\
& \left(e+\frac{v^{2}}{2}\right)_{t}+(p v)_{x}=0,
\end{aligned}
$$

where $\tau=1 / \rho$ is the specific volume with the density $\rho$, and $v, p, e$ are the velocity, the pressure, the internal energy, respectively; the other two gas dynamical variables are the temperature $\theta$ and the entropy $S$. For ideal polytropic gases, the system (1.1)-(1.3) is closed by the following constitutive relations:

$$
p \tau=R \theta, \quad e=c_{v} \theta, \quad p(\tau, S)=\kappa \tau^{-\gamma} e^{S / c_{v}},
$$

1991 Mathematics Subject Classification. Primary: 26B20,28C05, 35L65, 35B35; Secondary: 26B35, 26B12 35L67.

Key words and phrases. divergence-measure fields, normal traces, Gauss-Green theorem, product rule, Euler equations, gas dynamics, entropy solutions, stability, vacuum. 
where $c_{v}, R$, and $\kappa$ are positive constants, and $\gamma=1+c_{v} / R>1$. For isentropic gases, the Euler equations become

$$
\begin{aligned}
& \tau_{t}-v_{x}=0, \\
& v_{t}+p(\tau)_{x}=0,
\end{aligned}
$$

where $p(\tau)=\kappa \tau^{-\gamma}, \gamma>1$.

The main feature of nonlinear hyperbolic conservation laws, especially (1.1)(1.3), is that, no matter how smooth the initial data are, solutions may develop singularities and form shock waves in finite time. One may expect solutions in the space of functions of bounded variation. This is indeed the case by the Glimm theorem [15] which establishes that, when the initial data have sufficiently small total variation and stay away from vacuum for (1.1)-(1.3), there exists a global entropy solution in $B V$ satisfying the Clausius inequality:

$$
S_{t} \geq 0
$$

in the sense of distributions. However, when the initial data are large, still away from vacuum, the solutions may develop vacuum in finite time, even instantaneously as $t>0$. In this case, the specific volume $\tau=1 / \rho$ may then become a Radon measure or an $L^{1}$ function, rather than a function of bounded variation. This indicates that solutions of nonlinear hyperbolic conservation laws are generally either in $\mathcal{M}\left(\mathbb{R}_{+} \times \mathbb{R}^{n}\right)$, the space of signed Radon measures, or in $L^{p}\left(\mathbb{R}_{+} \times \mathbb{R}^{n}\right), 1 \leq p \leq \infty$. On the other hand, the fact that (1.1)-(1.3), and (1.7) hold in the sense of distributions implies, in particular, that the divergences of the fields $(\tau,-v),(v, p)$, $\left(e+v^{2} / 2, p v\right),(S, 0)$, in the $(t, x)$ variables, are Radon measures, in which the first three are the trivial null measure and the last one is a nonnegative measure as a consequence of the Schwartz Lemma [21]. This motivates our study of the extended divergence-measure fields (see Definition 1.1 below).

In this connection, we recall that Wagner [25] has proved that the well known Lagrangian transformation carries entropy solutions of the Euler equations in Eulerian coordinates to entropy solutions of (1.1)-(1.3), in a one-to-one and onto manner. However, since solutions of the first which contain vacuum are carried into solutions of (1.1)-(1.3) which are vector-valued measures, the concept of entropy solutions for the latter has to be strengthened. We will return to this point in Section 4.

Understanding more properties of $\mathcal{D} \mathcal{M}$-fields can advance our understanding of the behavior of entropy solutions (cf. $[5,6,7]$ ). One of the fundamental questions is whether the normal traces can still be defined and the Gauss-Green formula, i.e., integration by parts, still works for these extended fields, which are very weak.

We begin with the definition of $\mathcal{D} \mathcal{M}$-fields. For open sets $A, B \subset \mathbb{R}^{N}$, by the relation $A \Subset B$ we mean that the closure of $A, \bar{A}$, is a compact subset of $B$.

Definition 1.1. Let $D \subset \mathbb{R}^{N}$ be open. For $F \in L^{p}\left(D ; \mathbb{R}^{N}\right), 1 \leq p \leq \infty$, or $F \in \mathcal{M}\left(D ; \mathbb{R}^{N}\right)$, set

$$
|\operatorname{div} F|(D):=\sup \left\{\int_{D} \nabla \varphi \cdot F: \varphi \in C_{0}^{1}(D),|\varphi(x)| \leq 1, x \in D\right\} .
$$

For $1 \leq p \leq \infty$, we say that $F$ is an $L^{p}$-divergence-measure field over $D$, i.e., $F \in \mathcal{D} \bar{M}^{p}(D)$, if $F \in L^{p}\left(D ; \mathbb{R}^{N}\right)$ and

$$
\|F\|_{\mathcal{D M}^{p}(D)}:=\|F\|_{L^{p}\left(D ; \mathbb{R}^{N}\right)}+|\operatorname{div} F|(D)<\infty .
$$


We say that $F$ is an extended divergence-measure field over $D$, i.e., $F \in \mathcal{D M}^{e x t}(D)$, if $F \in \mathcal{M}\left(D ; \mathbb{R}^{N}\right)$ and

$$
\|F\|_{\mathcal{D M}^{e x t}(D)}:=|F|(D)+|\operatorname{div} F|(D)<\infty .
$$

If $F \in \mathcal{D} \mathcal{M}^{p}(D)$ for any open set $D \Subset \mathbb{R}^{N}$, then we say $F \in \mathcal{D} \mathcal{M}_{l o c}^{p}\left(\mathbb{R}^{N}\right)$; and, if $F \in \mathcal{D} \mathcal{M}^{\text {ext }}(D)$ for any open set $D \Subset \mathbb{R}^{N}$, we say $F \in \mathcal{D} \mathcal{M}_{\text {loc }}^{\text {ext }}\left(\mathbb{R}^{N}\right)$.

It is easy to check that these spaces under the norms (1.8) and (1.9), respectively, are Banach spaces. These spaces are larger than the $B V$-space. The establishment of the Gauss-Green theorem, traces, and other properties of BV functions in the middle of last century (see Federer [13]) has advanced significantly our understanding of solutions of nonlinear partial differential equations and nonlinear problems in calculus of variations, differential geometry, and other areas. A natural question is whether the $\mathcal{D} \mathcal{M}$-fields have similar properties, especially the normal traces and the Gauss-Green formula. At a first glance, it seems unclear.

EXAmple 1.1: The field $F(x, y)=\left(\frac{x}{x^{2}+y^{2}}, \frac{y}{x^{2}+y^{2}}\right)$ belongs to $\mathcal{D} \mathcal{M}_{l o c}^{1}\left(\mathbb{R}^{2}\right)$. As remarked in Whitney [26], for $\Omega=(0,1) \times(0,1)$,

$$
\int_{\Omega} \operatorname{div} F=0 \neq \int_{\partial \Omega} F \cdot \nu d \mathcal{H}^{1}=\frac{\pi}{2},
$$

if one understands $F \cdot \nu$ in the classical sense, which implies that the classical Gauss-Green theorem fails. In this paper, we succeed in using the neighborhood information via the Lipschitz deformation to develop a natural notion of normal traces, under which our generalized Gauss-Green theorem holds, even for $F \in$ $\mathcal{D} \mathcal{M}^{\text {ext }}(D)$.

EXAMPLE 1.2: For any $\mu \in \mathcal{M}(\mathbb{R})$ with finite total variation,

$$
F(x, y)=(d x \times \mu(y), 0) \in \mathcal{D} \mathcal{M}^{e x t}(I \times \mathbb{R}),
$$

for any bounded open interval $I \subset \mathbb{R}$. A non-trivial example of such fields is provided by the Riemann solutions of the Euler equations (1.1)-(1.3) for gas dynamics, which develop vacuum. See (4.12) below.

Some efforts have been made in generalizing the Gauss-Green Theorem. Some results for several situations can be found in Anzellotti [1] for an abstract formulation for $F \in L^{\infty}$, Rodrigues [20] for $F \in L^{2}$, and Ziemer [28] for a related problem for $\operatorname{div} F \in L^{1}$ (see also Baiocchi-Capelo [2], Brezzi-Fortin [4], and Ziemer [29]). In Chen-Frid [6], we observed an explicit way to calculate the normal traces for $F \in \mathcal{D} \mathcal{M}^{\infty}$ by the neighborhood information, under which a generalized GaussGreen theorem holds.

In this paper, motivated by various nonlinear problems from conservation laws, we propose a natural notion of normal traces by the neighborhood information via Lipschitz deformation under which a generalized Gauss-Green theorem is established for $F \in \mathcal{D} \mathcal{M}^{\text {ext }}(D)$ in Section 3, where our main results concerning extended divergence-measure fields are stated and proved, after establishing some auxiliary results in Section 2. In particular, we show an explicit way to calculate the normal traces over any deformable Lipschitz surface, suitable for applications, by using the neighborhood information of the fields near the surface and the level set function of the Lipschitz deformation surfaces. We also show a product rule for these extended fields. Their proofs require some refined properties of Radon measures and the Whitney extension theory, among others. 
In Section 4, we give an important application of the theory of $\mathcal{D} \mathcal{M}$-fields to the Euler equations (1.1)-(1.3) for gas dynamics and establish the uniqueness and stability of Riemann solutions of large oscillation that may contain two rarefaction waves and one contact discontinuity or vacuum states (i.e. measure solutions) in the class of entropy solutions, which may not belong to either $B V_{l o c}$ or $L^{\infty}$, without specific reference on the method of construction of the solutions. The proof, motivated by [11] and [7]-[9], is heavily based on our explicit approach to calculate normal traces over Lipschitz deformable surfaces, in the generalized GaussGreen theorem, and the product rule for $\mathcal{D} \mathcal{M}$-fields. The same arguments clearly also yield the uniqueness and stability of Riemann solutions in the class of entropy solutions for the Euler equations (1.5) and (1.6) for isentropic gas dynamics.

Before closing this introduction, we recall some correlated results. In DiPerna [11], a uniqueness theorem of Riemann solutions was first established for $2 \times 2$ systems in the class of entropy solutions in $L^{\infty} \cap B V_{l o c}$ with small oscillation. We also refer to Dafermos [10] for the stability of Lipschitz solutions for hyperbolic systems of conservation laws. In [8, 9], the uniqueness and stability of Riemann solutions of large oscillation without vacuum (possibly containing shocks) was proved for the $3 \times 3$ Euler equations, in the class of entropy solutions in $L^{\infty} \cap B V_{l o c}$ which stay away from vacuum. Another related connection is the recent results on the $L^{1}$-stability of the solutions in $L^{\infty} \cap B V$ obtained either by the Glimm scheme [15], the wave front-tracking method, the vanishing viscosity method, or more generally satisfying an additional regularity, with small total variation in $x$ uniformly for all $t>0$ (see the recent references cited in Bianchini-Bressan [3] and Dafermos [10]).

\section{Radon Measures and the Whitney Extension}

In this section, we establish some auxiliary properties about Radon measures and the Whitney extension of Lipschitz continuous functions, which are required for our analysis on the extended divergence-measure vector fields in Section 3. We begin with some properties about Radon measures. Let $\Omega, D \subset \mathbb{R}^{N}$ be open. For $\mu^{\epsilon}, \mu \in \mathcal{M}(\Omega)$, we denote $\mu^{\epsilon} \rightarrow \mu$ the weak convergence of $\mu^{\epsilon}$ to $\mu$ in $\mathcal{M}(\Omega)$. The next three lemmas are standard, but we include their proofs for completeness

Lemma 2.1. Let $\mu^{\varepsilon}, \mu \in \mathcal{M}(\Omega)$ be signed Radon measures over $\Omega$ with $\mu^{\varepsilon} \rightarrow \mu$. Then

$$
|\mu|(\Omega) \leq \liminf _{\varepsilon \rightarrow 0}\left|\mu^{\varepsilon}\right|(\Omega) .
$$

This can be seen as follows: For any $\phi \in C_{0}(\Omega),|\phi| \leq 1$,

$$
\langle\mu, \phi\rangle=\lim _{\varepsilon \rightarrow 0}\left\langle\mu^{\varepsilon}, \phi\right\rangle \leq \liminf _{\varepsilon \rightarrow 0}\left|\mu^{\varepsilon}\right|(\Omega) .
$$

Lemma 2.2. Let $\mu^{\varepsilon}, \mu \in \mathcal{M}(\Omega)$ be such that

$$
\mu^{\varepsilon} \rightarrow \mu, \quad \lim _{\varepsilon \rightarrow 0}\left|\mu^{\varepsilon}\right|(\Omega)=|\mu|(\Omega) .
$$

Then, for every open set $A \subset \Omega$,

$$
|\mu|(\bar{A} \cap \Omega) \geq \limsup _{\varepsilon \rightarrow 0}\left|\mu^{\varepsilon}\right|(\bar{A} \cap \Omega) .
$$

In particular, if $|\mu|(\partial A \cap \Omega)=0$, then

$$
|\mu|(A)=\lim _{\varepsilon \rightarrow 0}\left|\mu^{\varepsilon}\right|(A) .
$$


Proof. Set $B=\Omega-\bar{A}$, which is open. Then

$$
|\mu|(A) \leq \liminf _{\varepsilon \rightarrow 0}\left|\mu^{\varepsilon}\right|(A), \quad|\mu|(B) \leq \liminf _{\varepsilon \rightarrow 0}\left|\mu^{\varepsilon}\right|(B) .
$$

On the other hand,

$$
\begin{aligned}
|\mu|(\bar{A} \cap \Omega)+|\mu|(B) & =|\mu|(\Omega)=\lim _{\varepsilon \rightarrow 0}\left|\mu^{\varepsilon}\right|(\Omega) \\
& \geq \limsup \left|\mu^{\varepsilon}\right|(\bar{A} \cap \Omega)+\liminf \left|\mu^{\varepsilon}\right|(B) \\
& \geq \limsup \left|\mu^{\varepsilon}\right|(\bar{A} \cap \Omega)+|\mu|(B),
\end{aligned}
$$

which yields the desired result.

Lemma 2.3. Let $\omega^{\varepsilon}$ be a sequence of positive symmetric mollifiers in $\mathbb{R}^{N}$ and $\mu \in \mathcal{M}(\Omega)$. Set $\mu^{\varepsilon}=\mu * \omega^{\varepsilon}$, the mollified measures. Then, for any open set $A \Subset \Omega$ with $|\mu|(\partial A)=0$,

$$
|\mu|(A)=\lim _{\varepsilon \rightarrow 0}\left|\mu^{\varepsilon}\right|(A) .
$$

Proof. Since $\mu^{\varepsilon} \rightarrow \mu$, Lemma 2.1 implies

$$
|\mu|(A) \leq \liminf _{\varepsilon \rightarrow 0}\left|\mu^{\varepsilon}\right|(A) .
$$

Notice that, for any $g \in C_{0}(A),|g| \leq 1$,

$$
\left\langle\mu^{\varepsilon}, g\right\rangle=\left\langle\mu, g^{\varepsilon}\right\rangle
$$

where $g^{\varepsilon}=g * \omega^{\varepsilon}$. Since $\left|g^{\varepsilon}\right| \leq 1$ and $\operatorname{spt}\left(g^{\varepsilon}\right) \subset A_{\varepsilon}:=\{x: \operatorname{dist}(x, A) \leq \varepsilon\} \subset \Omega$ for $\varepsilon \ll 1$, then

which implies

$$
\left|\left\langle\mu^{\varepsilon}, g\right\rangle\right| \leq|\mu|\left(A_{\varepsilon}\right)
$$

$$
\left|\mu^{\varepsilon}\right|(A) \leq|\mu|\left(A_{\varepsilon}\right)
$$

Hence,

which yields

$$
\limsup _{\varepsilon \rightarrow 0}\left|\mu^{\varepsilon}\right|(A) \leq \lim _{\varepsilon \rightarrow 0}|\mu|\left(A_{\varepsilon}\right)=|\mu|(\bar{A})
$$

since $|\mu|(\partial A)=0$.

$$
\limsup _{\varepsilon \rightarrow 0}\left|\mu^{\varepsilon}\right|(A) \leq|\mu|(A)
$$

In particular, Lemma 2.3 indicates that, if $\mu \geq 0$ and $\mu(\partial A)=0$, then

$$
\mu(A)=\lim _{\varepsilon \rightarrow 0} \mu^{\varepsilon}(A) .
$$

Proposition 2.1. Let $D \subset \mathbb{R}^{N}$ be open, $\mu \in \mathcal{M}(D)$, and $\mu^{\varepsilon}=\mu * \omega^{\varepsilon}$. Let $\Omega \Subset D$ be open and $|\mu|(\partial \Omega)=0$. Then, for any $\phi \in C(D)$,

$$
\lim _{\varepsilon \rightarrow 0}\left\langle\mu^{\varepsilon}, \phi \chi_{\Omega}\right\rangle=\left\langle\mu, \phi \chi_{\Omega}\right\rangle \text {. }
$$

Proof. Write

$$
\mu^{\varepsilon}=\mu^{\varepsilon+}-\mu^{\varepsilon-}
$$

where $\mu^{\varepsilon \pm}=\mu^{ \pm} * \omega^{\varepsilon}$ are nonnegative measures. The condition $|\mu|(\partial \Omega)=0$ implies

$$
\mu^{ \pm}(\partial \Omega)=0
$$

From Lemma 2.3, we have

$$
\lim _{\varepsilon \rightarrow 0} \mu^{\varepsilon \pm}(\Omega)=\mu^{ \pm}(\Omega)
$$


Hence

$$
\lim _{\varepsilon \rightarrow 0} \mu^{\varepsilon}(\Omega)=\lim _{\varepsilon \rightarrow 0} \mu^{\varepsilon+}(\Omega)-\lim _{\varepsilon \rightarrow 0} \mu^{\varepsilon-}(\Omega)=\mu^{+}(\Omega)-\mu^{-}(\Omega)=\mu(\Omega) .
$$

Let $A$ be open with $\Omega \Subset A \Subset D$. Then, for $\phi \in C(D)$ and $\delta>0$, we may construct a partition of $\mathbb{R}^{N}$ by means of parallelepipeds:

$$
Q_{\alpha}=\left[x_{1}^{\alpha_{1}}, x_{1}^{\alpha_{1}+1}\right] \times \cdots \times\left[x_{N}^{\alpha_{N}}, x_{N}^{\alpha_{N}+1}\right], \quad \alpha=\left(\alpha_{1}, \cdots, \alpha_{N}\right) \in \mathbb{Z}^{N},
$$

such that

$$
\begin{aligned}
& \cup_{Q_{\alpha} \cap \Omega \neq \emptyset} Q_{\alpha} \subset A, \\
& |\mu|\left(\partial Q_{\alpha} \cap D\right)=0, \quad \alpha \in \mathbb{Z}^{N},
\end{aligned}
$$

and

$$
|\phi(x)-\phi(y)|<\delta, \quad x, y \in Q_{\alpha} .
$$

Let $a_{\alpha}=\phi\left(x_{\alpha}^{*}\right)$ for some $x_{\alpha}^{*} \in Q_{\alpha}$ if $Q_{\alpha} \cap \Omega \neq \emptyset$, and $a_{\alpha}=0$, otherwise. Then

$$
\left\langle\mu^{\varepsilon}, \phi \chi_{\Omega}\right\rangle=\left\langle\mu^{\varepsilon}, \sum a_{\alpha} \chi_{Q_{\alpha} \cap \Omega}\right\rangle+\left\langle\mu^{\varepsilon},\left(\phi-\sum a_{\alpha} \chi_{Q_{\alpha}}\right) \chi_{\Omega}\right\rangle,
$$

and

$$
\begin{aligned}
\limsup _{\varepsilon \rightarrow 0}\left\langle\mu^{\varepsilon}, \phi \chi_{\Omega}\right\rangle & \leq\left\langle\mu, \sum a_{\alpha} \chi_{Q_{\alpha} \cap \Omega}\right\rangle+\delta|\mu|(A) \\
& \leq\left\langle\mu, \phi \chi_{\Omega}\right\rangle+2 \delta|\mu|(A)
\end{aligned}
$$

Analogously,

$$
\liminf _{\varepsilon \rightarrow 0}\left\langle\mu^{\varepsilon}, \phi \chi_{\Omega}\right\rangle \geq\left\langle\mu, \phi \chi_{\Omega}\right\rangle-2 \delta|\mu|(A) .
$$

Since $\delta>0$ is arbitrary, we complete the proof.

We now discuss some concepts and facts about the extension of Lipschitz continuous functions defined on a closed set $C \subset \mathbb{R}^{N}$ to $\mathbb{R}^{N}$, following the theory set forth by Whitney [27] (see also [23]), which play an important role in Section 3.

Let $k$ be a nonnegative integer and $\gamma \in(k, k+1]$. We say that a function $f$, defined on $C$, belongs to $\operatorname{Lip}(\gamma, C)$ if there exist functions $f^{(j)}, 0 \leq|j| \leq k$, defined on $C$, with $f^{(0)}=f$, such that, if

$$
f^{(j)}(x)=\sum_{|j+l| \leq k} \frac{f^{(j+l)}(y)}{l !}(x-y)^{l}+R_{j}(x, y),
$$

then

$$
\left\{\begin{array}{l}
\left|f^{(j)}(x)\right| \leq M, \\
\left|R_{j}(x, y)\right| \leq M|x-y|^{\gamma-|j|}, \quad \text { for any } x, y \in C,|j| \leq k .
\end{array}\right.
$$

Here $j$ and $l$ denote multi-indices $j=\left(j_{1}, \cdots, j_{N}\right)$ and $l=\left(l_{1}, \cdots, l_{N}\right)$ with $j !=$ $j_{1} ! \cdots j_{N} !,|j|=j_{1}+j_{2}+\cdots+j_{N}$, and $x^{l}=x_{1}^{l_{1}} x_{2}^{l_{2}} \cdots x_{N}^{l_{N}}$. By an element of $\operatorname{Lip}(\gamma, C)$ we mean the collection $\left\{f^{(j)}(x)\right\}_{|j| \leq k}$. The norm of an element in $\operatorname{Lip}(\gamma, C)$ is defined as the smallest $M$ for which the inequality (2.1) holds. We notice that $\operatorname{Lip}(\gamma, C)$ with this norm is a Banach space. For the case $C=\mathbb{R}^{N}$, since the functions $f^{(j)}$ are determined by $f^{(0)}$, this collection is then identified with $f^{(0)}$.

The Whitney extension of order $k$ is defined as follows. Let $\left\{f^{(j)}\right\}_{|j| \leq k}$ be an element of $\operatorname{Lip}(\gamma, C)$. The linear mapping $\mathcal{E}_{k}: \operatorname{Lip}(\gamma, C) \rightarrow \operatorname{Lip}\left(\gamma, \mathbb{R}^{N}\right)$ assigns 
to any such collection a function $\mathcal{E}_{k}\left(f^{(j)}\right)$ defined on $\mathbb{R}^{N}$ which is an extension of $f^{(0)}=f$ to $\mathbb{R}^{N}$. The definition of $\mathcal{E}_{k}$ is the following:

$$
\left\{\begin{array}{l}
\mathcal{E}_{0}(f)(x)=f(x), \quad x \in C, \\
\mathcal{E}_{0}(f)(x)=\sum_{i} f\left(p_{i}\right) \varphi_{i}(x), \quad x \in \mathbb{R}^{N}-C,
\end{array}\right.
$$

and, for $k>0$,

$$
\left\{\begin{array}{l}
\mathcal{E}_{k}\left(f^{(j)}\right)(x)=f^{(0)}(x), \quad x \in C, \\
\mathcal{E}_{k}\left(f^{(j)}\right)(x)=\sum_{i}^{\prime} P\left(x, p_{i}\right) \varphi_{i}(x), \quad x \in \mathbb{R}^{N}-C .
\end{array}\right.
$$

Here $P(x, y)$ denotes the polynomial in $x$, which is the Taylor expansion of $f$ about the point $y \in C$ :

$$
P(x, y)=\sum_{|l| \leq k} \frac{f^{(l)}(y)(x-y)^{l}}{l !}, \quad x \in \mathbb{R}^{N}, y \in C .
$$

The functions $\varphi_{i}$ form a partition of unity of $\mathbb{R}^{N}-C$ with the following properties:

(i) $\operatorname{spt}\left(\varphi_{i}\right) \subset Q_{i}$ where $Q_{i}$ is a cube with edges parallel to the coordinate axes and

$$
c_{1} \operatorname{diam}\left(Q_{i}\right) \leq \operatorname{dist}\left(Q_{i}, C\right) \leq c_{2} \operatorname{diam}\left(Q_{i}\right),
$$

for certain positive constants $c_{1}$ and $c_{2}$ independent of $C$;

(ii) each point of $\mathbb{R}^{N}-C$ is contained in at most $N_{0}$ cubes $Q_{i}$, for certain number $N_{0}$ depending only on the dimension $N$;

(iii) the derivatives of $\varphi_{i}$ satisfy

$$
\left|\partial_{x_{1}}^{\alpha_{1}} \cdots \partial_{x_{N}}^{\alpha_{N}} \varphi_{i}(x)\right| \leq A_{\alpha}\left(\operatorname{diam} Q_{i}\right)^{-|\alpha|} .
$$

Here $p_{i} \in C$ is such that $\operatorname{dist}\left(Q_{i}, C\right)=\operatorname{dist}\left(p_{i}, Q_{i}\right),|\alpha|=\alpha_{1}+\cdots+\alpha_{N}$, and the symbol $\sum^{\prime}$ indicates that the summation is taken only over those cubes whose distances to $C$ are not greater than one.

The following theorem, whose proof can also be found in [23], is due to Whitney [27].

Theorem 2.1. Suppose that $k$ is a nonnegative integer, $\gamma \in(k, k+1]$, and $C$ is a closed set. Then the mapping $\mathcal{E}_{k}$ is a continuous linear mapping from $\operatorname{Lip}(\gamma, C)$ to Lip $\left(\gamma, \mathbb{R}^{N}\right)$ which defines an extension of $f^{(0)}$ to $\mathbb{R}^{N}$, and the norm of this mapping has a bound independent of $C$.

We will need the following proposition, which seems to be of interest in itself and is useful in establishing the generalized Gauss-Green theorem in Section 3.

Proposition 2.2. Let $C$ be a closed set in $\mathbb{R}^{N}$ and

$$
C_{\delta}:=\left\{x \in \mathbb{R}^{N}: \operatorname{dist}(x, C) \leq \delta\right\}, \quad \text { for } \delta>0 .
$$

Let $\mathcal{E}_{k}: \operatorname{Lip}(\gamma, C) \rightarrow \operatorname{Lip}\left(\gamma, \mathbb{R}^{N}\right)$ with $\gamma \in(k, k+1]$ be the Whitney extension of order $k$. Then, for any $\phi \in \operatorname{Lip}\left(\gamma, \mathbb{R}^{N}\right)$ and any $\gamma^{\prime} \in(k, \gamma)$,

$$
\left\|\mathcal{E}_{k}(\phi \mid C)-\phi\right\|_{L i p\left(\gamma^{\prime}, C_{\delta}\right)} \rightarrow 0, \quad \text { as } \delta \rightarrow 0 .
$$

Proof. We will prove the proposition in detail only for the cases $k=0$ and $k=1$ since the case $k>1$ can then be obtained by induction. 
For $k=0, \mathcal{E}_{0}$ is given by $\mathcal{E}_{0}(f)(x)=f(x)$ if $x \in C$, and

$$
\mathcal{E}_{0}(f)(x)=\sum_{i=1}^{\infty} f\left(p_{i}\right) \varphi_{i}(x), \quad \text { if } x \in \mathbb{R}^{N}-C,
$$

where $p_{i}$ and $\varphi_{i}$ are as above. Now, for any $\phi \in \operatorname{Lip}\left(\gamma, \mathbb{R}^{N}\right)$, we have

$$
\mathcal{E}_{0}(\phi \mid C)(x)-\phi(x)=\sum_{i=1}^{\infty}\left(\phi\left(p_{i}\right)-\phi(x)\right) \varphi_{i}(x) .
$$

Clearly,

$$
\sup _{x \in C_{\delta}}\left|\mathcal{E}_{0}(\phi \mid C)(x)-\phi(x)\right| \leq c \sup _{\substack{y \in C, x \in C_{\delta} \\|x-y| \leq c_{0} \delta}}|\phi(y)-\phi(x)| \rightarrow 0, \text { as } \delta \rightarrow 0 .
$$

Here and in what follows in this proof $c_{0}$ and $c$ are positive constants, which do not depend on either $\delta>0$ or $C$, whose values may change at each appearance.

Set $g=\mathcal{E}_{0}(\phi \mid C)-\phi$. We now show that

$$
|g(x)-g(y)| \leq M(\delta)|x-y|^{\gamma^{\prime}}, \quad x, y \in C_{\delta},
$$

where $M(\delta) \rightarrow 0$ as $\delta \rightarrow 0$. Indeed,

$$
g(x)-g(y)=\sum_{x \in Q_{i}}\left(\phi\left(p_{i}\right)-\phi(x)\right) \varphi_{i}(x)-\sum_{y \in Q_{i}}\left(\phi\left(p_{i}\right)-\phi(y)\right) \varphi_{i}(y) .
$$

We split each of these sums into two, respectively:

$$
\sum_{x \in Q_{i}}=\sum_{\substack{x \in Q_{i} \\ y \notin Q_{i}}}+\sum_{\substack{x \in Q_{i} \\ y \in Q_{i}}}, \quad \sum_{y \in Q_{i}}=\sum_{\substack{y \in Q_{i} \\ x \notin Q_{i}}}+\sum_{\substack{x \in Q_{i} \\ y \in Q_{i}}},
$$

and denote

$$
\sum^{0}=\sum_{\substack{x \in Q_{i} \\ y \in Q_{i}}}, \quad \sum^{1}=\sum_{\substack{x \in Q_{i} \\ y \notin Q_{i}}}, \quad \sum^{2}=\sum_{\substack{y \in Q_{i} \\ x \notin Q_{i}}}
$$

We have

$$
\begin{aligned}
& \sum^{0}\left(\left(\phi\left(p_{i}\right)-\phi(x)\right) \varphi_{i}(x)-\left(\phi\left(p_{i}\right)-\phi(y)\right) \varphi_{i}(y)\right) \\
& =\sum^{0}(\phi(y)-\phi(x)) \varphi_{i}(x)+\sum^{0}\left(\phi\left(p_{i}\right)-\phi(y)\right)\left(\varphi_{i}(x)-\varphi_{i}(y)\right) .
\end{aligned}
$$

Since, if $x, y \in Q_{i} \cap C_{\delta},|x-y| \leq c_{0} \delta$, for a given constant $c_{0}>0$, we obtain, for the first sum,

$$
\left|\sum^{0}(\phi(x)-\phi(y)) \varphi_{i}(x)\right| \leq c \delta^{\gamma-\gamma^{\prime}}|x-y|^{\gamma^{\prime}},
$$

and, for the second sum,

$$
\begin{gathered}
\left|\sum^{0}\left(\phi\left(p_{i}\right)-\phi(y)\right)\left(\varphi_{i}(x)-\varphi_{i}(y)\right)\right| \leq c \sum^{0}\left|p_{i}-y\right|^{\gamma}\left(\operatorname{diam} Q_{i}\right)^{-1}|x-y| \\
\leq c|x-y|^{\gamma} \leq c \delta^{\gamma-\gamma^{\prime}}|x-y|^{\gamma^{\prime}} .
\end{gathered}
$$

Now, for $\sum^{1}$, we have

$$
\sum^{1}\left(\phi\left(p_{i}\right)-\phi(x)\right) \varphi_{i}(x)=\sum^{1}\left(\phi\left(p_{i}\right)-\phi(x)\right)\left(\varphi_{i}(x)-\varphi_{i}\left(q_{i}\right)\right),
$$


with $q_{i}=\partial Q_{i} \cap[x, y]$, where $[x, y]$ is the straight line segment connecting $x$ to $y$. Therefore,

$$
\begin{gathered}
\sum^{1}\left|\phi\left(p_{i}\right)-\phi(x)\right|\left|\varphi_{i}(x)-\varphi_{i}\left(q_{i}\right)\right| \leq c \sum^{1}\left|p_{i}-x\right|^{\gamma}\left(\operatorname{diam} Q_{i}\right)^{-1}\left|x-q_{i}\right| \\
\leq c \sum^{1}\left|x-q_{i}\right|^{\gamma} \leq c \delta^{\gamma-\gamma^{\prime}}|x-y|^{\gamma^{\prime}} .
\end{gathered}
$$

The sum $\sum^{2}$ is treated similarly. We have then proved (2.6) which, together with (2.5), gives (2.3) for $k=0$.

For $k=1$, we have $\mathcal{E}_{1}(f)(x)=f(x)$ for $x \in C$, and

$$
\mathcal{E}_{1}(f)(x)=\sum_{i=1}^{\infty} P_{f}\left(x, p_{i}\right) \varphi_{i}(x), \quad \text { for } \quad x \in \mathbb{R}^{N}-C,
$$

where

$$
P_{f}(x, y)=f(y)+\sum_{j=1}^{N} \partial_{x_{j}} f(y)\left(x_{j}-y_{j}\right),
$$

and the functions $\varphi_{i}$, conveniently renumbered together with the $Q_{i}$ containing $\operatorname{spt}\left(\varphi_{i}\right)$, satisfy $\operatorname{dist}\left(Q_{i}, C\right) \leq 1$. Since $\phi \in \operatorname{Lip}\left(\gamma, \mathbb{R}^{N}\right), \gamma>1$, we clearly have

$$
(\phi \mid C)^{j}(y)=\partial_{x_{j}} \phi(y), \quad \text { for } y \in C .
$$

Hence

$$
\mathcal{E}_{1}(\phi \mid C)(x)-\phi(x)=\sum_{i=1}^{\infty}\left(P_{\phi}\left(x, p_{i}\right)-\phi(x)\right) \varphi_{i}(x) .
$$

Setting $g_{1}=\mathcal{E}_{1}(\phi \mid C)-\phi$, we have

$$
\begin{aligned}
\left|g_{1}(x)\right| & \leq \sum_{i=1}^{\infty}\left|P_{\phi}\left(x, p_{i}\right)-\phi(x)\right|\left|\varphi_{i}(x)\right| \\
& \leq c \sum_{x \in Q_{i}}\left|x-p_{i}\right|^{\gamma}\left|\varphi_{i}(x)\right| \leq c \delta^{\gamma} \rightarrow 0, \quad \text { as } \delta \rightarrow 0 .
\end{aligned}
$$

Also,

$$
\begin{aligned}
\partial_{x_{k}} g_{1}(x) & =\sum_{x \in Q_{i}} \partial_{x_{k}}\left(P_{\phi}\left(x, p_{i}\right)-\phi(x)\right) \varphi_{i}(x)+\sum_{x \in Q_{i}}\left(P_{\phi}\left(x, p_{i}\right)-\phi(x)\right) \partial_{x_{k}} \varphi_{i}(x) \\
& =h_{1}(x)+h_{2}(x)
\end{aligned}
$$

where

$h_{1}(x):=\sum_{x \in Q_{i}}\left(\partial_{x_{k}} \phi\left(p_{i}\right)-\partial_{x_{k}} \phi(x)\right) \varphi_{i}(x), \quad h_{2}(x):=\sum_{x \in Q_{i}}\left(P_{\phi}\left(x, p_{i}\right)-\phi(x)\right) \partial_{x_{k}} \varphi_{i}(x)$.

Hence,

$$
\begin{aligned}
\left|\partial_{x_{k}} g_{1}(x)\right| & \leq c \sum_{x \in Q_{i}}\left|p_{i}-x\right|^{\gamma-1}+c \sum_{x \in Q_{i}}\left|x-p_{i}\right|^{\gamma} \operatorname{diam}\left(Q_{i}\right)^{-1} \\
& \leq c \delta^{\gamma-1} \rightarrow 0, \quad \text { as } \delta \rightarrow 0 .
\end{aligned}
$$

We now show

$$
\left|\partial_{x_{k}} g_{1}(x)-\partial_{x_{k}} g_{1}(y)\right| \leq M(\delta)|x-y|^{\gamma^{\prime}-1}
$$

with $M(\delta) \rightarrow 0$ as $\delta \rightarrow 0$. First we obtain

$$
\left|h_{1}(x)-h_{1}(y)\right| \leq M(\delta)|x-y|^{\gamma^{\prime}-1}, \quad \text { with } M(\delta) \rightarrow 0, \quad \text { as } \delta \rightarrow 0,
$$


exactly as in the case $k=0$. Now,

$$
\begin{aligned}
h_{2}(x)-h_{2}(y) & =\sum_{x \in Q_{i}}\left(P_{\phi}\left(x, p_{i}\right)-\phi(x)\right) \partial_{x_{k}} \varphi_{i}(x)-\sum_{x \in Q_{i}}\left(P_{\phi}\left(x, p_{i}\right)-\phi(y)\right) \partial_{x_{k}} \varphi_{i}(y) \\
& =\sum_{x \in Q_{i}} R\left(x, p_{i}\right) \partial_{x_{k}} \varphi_{i}(x)-\sum_{y \in Q_{i}} R\left(y, p_{i}\right) \partial_{x_{k}} \varphi_{i}(y),
\end{aligned}
$$

where we put $R(x, y)=P_{\phi}(x, y)-\phi(x)$. Again, we split each of the last two sums above into two, respectively:

$$
\sum_{x \in Q_{i}}=\sum^{0}+\sum^{1}, \quad \sum_{y \in Q_{i}}=\sum^{0}+\sum^{2},
$$

as in the first part of the proof, and compute

$$
\begin{aligned}
& \left|\sum^{0} R\left(x, p_{i}\right) \partial_{x_{k}} \varphi_{i}(x)-\sum^{0} R\left(y, p_{i}\right) \partial_{x_{k}} \varphi_{i}(y)\right| \\
& \leq\left|\sum^{0}\left(R\left(x, p_{i}\right)-R\left(y, p_{i}\right)\right) \partial_{x_{k}} \varphi_{i}(x)\right|+\left|\sum^{0} R\left(y, p_{i}\right)\left(\partial_{x_{k}} \varphi_{i}(y)-\partial_{x_{k}} \varphi_{i}(x)\right)\right| \\
& \leq c \sum^{0}|x-y|^{\gamma}\left(\operatorname{diam} Q_{i}\right)^{-1}+c \sum^{0}\left|y-p_{i}\right|^{\gamma}\left(\operatorname{diam} Q_{i}\right)^{-2}|x-y| \\
& \leq c \delta^{\gamma-\gamma^{\prime}}|x-y|^{\gamma^{\prime}-1} .
\end{aligned}
$$

For the remaining sums, we have

$$
\begin{aligned}
& \left|\sum^{1} R\left(x, p_{i}\right) \partial_{x_{k}} \varphi_{i}(x)\right|=\left|\sum^{1} R\left(x, p_{i}\right)\left(\partial_{x_{k}} \varphi_{i}(x)-\partial_{x_{k}} \varphi_{i}\left(q_{i}\right)\right)\right| \\
& \leq c \sum^{1}\left|x-p_{i}\right|^{\gamma}\left(\operatorname{diam} Q_{i}\right)^{-2}\left|x-q_{i}\right| \\
& \leq c \delta^{\gamma-\gamma^{\prime}}|x-y|^{\gamma^{\prime}-1},
\end{aligned}
$$

where $q_{i}$ is as that in the first part of the proof; and the sum $\sum^{2} R\left(y, p_{i}\right) \partial_{x_{k}} \varphi_{i}(y)$ is treated similarly. This concludes the proof in the case $k=1$. As indicated above, the case $k>1$ follows similarly by induction.

\section{Normal Traces and Generalized Gauss-Green Theorem}

In this section, we prove our main results concerning extended $\mathcal{D} \mathcal{M}$-fields, including a generalized Gauss-Green theorem, a new notion of normal traces, and a product rule for $\mathcal{D} \mathcal{M}$-fields. We begin with the definition of deformable Lipschitz boundaries.

Definition 3.1. Let $\Omega \subset \mathbb{R}^{N}$ be an open bounded subset. We say that $\partial \Omega$ is a deformable Lipschitz boundary, provided that

(i) $\forall x \in \partial \Omega, \exists r>0$ and a Lipschitz map $\gamma: \mathbb{R}^{N-1} \rightarrow \mathbb{R}$ such that, after rotating and relabeling coordinates if necessary,

$$
\Omega \cap Q(x, r)=\left\{y \in \mathbb{R}^{N}: \gamma\left(y_{1}, \cdots, y_{N-1}\right)<y_{N}\right\} \cap Q(x, r),
$$

where $Q(x, r)=\left\{y \in \mathbb{R}^{N}:\left|x_{i}-y_{i}\right| \leq r, i=1, \cdots, N\right\}$;

(ii) $\exists \Psi: \partial \Omega \times[0,1] \rightarrow \bar{\Omega}$ such that $\Psi$ is a homeomorphism bi-Lipschitz over its image and $\Psi(\omega, 0)=\omega$ for all $\omega \in \partial \Omega$. The map $\Psi$ is called a Lipschitz deformation of the boundary $\partial \Omega$.

The following lemma is a direct corollary of the boundedness of $F$ and $\operatorname{div} F$ over $\Omega$ as Radon measures. Since the theory of $\mathcal{D} \mathcal{M}^{\infty}$-fields has been addressed in [5], henceforth we focus on $\mathcal{D} \mathcal{M}^{*}$-fields, where $*$ stands for either $p \in[1, \infty)$ or ext. 
Lemma 3.1. Let $F \in \mathcal{D M}^{*}(\Omega)$ with $\Omega$ an open set whose boundary $\partial \Omega$ has a Lipschitz deformation $\Psi$ with $\partial \Omega_{s}=\Psi_{s}(\partial \Omega), s \in[0,1]$. Then there exists a countable set $\mathcal{T} \subset(0,1)$ such that

$$
|F|\left(\partial \Omega_{s}\right)=|\operatorname{div} F|\left(\partial \Omega_{s}\right)=0, \quad \text { for any } s \in(0,1)-\mathcal{T} .
$$

We now establish the generalized Gauss-Green theorem for $\mathcal{D M}^{*}$-fields, by introducing a suitable definition of normal traces over the boundary $\partial \Omega$ of a bounded open set $\Omega$ with Lipschitz deformable boundary.

Theorem 3.1 (Generalized Gauss-Green Theorem for $\mathcal{D} \mathcal{M}^{*}$-fields). Assume $F \in$ $\mathcal{D M}^{*}(\Omega)$. Let $\Omega \subset \mathbb{R}^{N}$ is a bounded open set with Lipschitz deformable boundary. Then there exists a continuous linear functional $\left.F \cdot \nu\right|_{\partial \Omega}$ over Lip $(\gamma, \partial \Omega), \gamma>1$, such that, for any $\phi \in \operatorname{Lip}\left(\gamma, \mathbb{R}^{N}\right)$,

$$
\left\langle\left. F \cdot \nu\right|_{\partial \Omega}, \phi\right\rangle=\int_{\Omega} \phi \operatorname{div} F+\int_{\Omega} \nabla \phi \cdot F .
$$

Let $h: \mathbb{R}^{N} \rightarrow \mathbb{R}$ be the level set function of $\partial \Omega_{s}$, that is,

$$
h(x):= \begin{cases}0, & \text { for } x \in \mathbb{R}^{N}-\bar{\Omega}, \\ 1, & \text { for } x \in \Omega-\Psi(\partial \Omega \times[0,1]), \\ s, & \text { for } x \in \partial \Omega_{s}, 0 \leq s \leq 1 .\end{cases}
$$

Then, if $F \in \mathcal{D M}^{p}(\Omega), 1 \leq p<\infty$,

$$
\begin{aligned}
\left\langle\left. F \cdot \nu\right|_{\partial \Omega}, \psi\right\rangle & =-\lim _{s \rightarrow 0} \frac{1}{s} \int_{\Psi(\partial \Omega \times(0, s))} \mathcal{E}(\psi) \nabla h \cdot F d x \\
& =-\lim _{s \rightarrow 0} \frac{1}{s} \int_{\Psi(\partial \Omega \times(0, s))} \mathcal{E}(\psi)|\nabla h| F \cdot \nu d x
\end{aligned}
$$

for any $\psi \in \operatorname{Lip}(\partial \Omega)$, where $\mathcal{E}(\psi)$ is any Lipschitz extension of $\psi$ to all $\mathbb{R}^{N}$ and $\nu: \Psi(\partial \Omega \times[0,1]) \rightarrow \mathbb{R}^{N}$ is such that $\nu(x)$ is the unit outer normal to $\partial \Omega_{s}$ at $x \in \partial \Omega_{s}$, defined for a.e. $x \in \Psi(\partial \Omega \times[0,1])$. Formula (3.2) also holds if $F \in \mathcal{D} \mathcal{M}^{\text {ext }}(\Omega)$, for any $\psi \in \operatorname{Lip}(\gamma, \partial \Omega), \gamma>1$, and $\mathcal{E}(\psi) \in \operatorname{Lip}\left(\gamma, \partial \mathbb{R}^{N}\right)$ any extension of $\psi$ to $\mathbb{R}^{N}$, provided that the set of non-Lebesgue points of $\nabla h(x)$ on $\Psi(\partial \Omega \times(0,1))$ has $|F|$-measure zero. Finally, for $F \in \mathcal{D M}^{p}(\Omega)$ with $1<p<\infty,\left.F \cdot \nu\right|_{\partial \Omega}$ can be extended to a continuous linear functional over $W^{1-1 / p, p}(\partial \Omega) \cap C(\partial \Omega)$.

Proof. We divide the proof into four steps.

Step 1. We first treat the more general case $F \in \mathcal{D M}^{\text {ext }}(\Omega)$. For $\psi \in \operatorname{Lip}(\gamma, \partial \Omega)$, let $\mathcal{E}(\psi)$ be the Whitney extension of $\psi$ of order $k=1$. Then, by the classical Gauss-Green formula, we have

$$
\int_{\partial \Omega} F^{\varepsilon} \cdot \nu \psi d \mathcal{H}^{N-1}=\int_{\Omega} \mathcal{E}(\psi) \operatorname{div} F^{\varepsilon}+\int_{\Omega} \nabla \mathcal{E}(\psi) \cdot F^{\varepsilon} .
$$

where $F^{\varepsilon}=F * \omega_{\varepsilon}$ with the standard mollified sequence $\omega_{\varepsilon}$.

To begin with, we first focus on $F \in \mathcal{D} \mathcal{M}^{\text {ext }}(D)$, for $\Omega \Subset D \Subset \mathbb{R}^{N}$, satisfying

$$
|F|(\partial \Omega)=|\operatorname{div} F|(\partial \Omega)=0 \text {. }
$$

The right-hand side of (3.4) defines a uniformly bounded family of continuous linear operators $l^{\varepsilon}$ over $\operatorname{Lip}(\gamma, \partial \Omega)$. Moreover, for each $\psi \in \operatorname{Lip}(\gamma, \partial \Omega)$, the limit 
$\lim _{\varepsilon \rightarrow 0} l^{\varepsilon}(\psi)$ exists and equals

$$
\int_{\Omega} \mathcal{E}(\psi) \operatorname{div} F+\int_{\Omega} \nabla \mathcal{E}(\psi) \cdot F
$$

as a consequence of (3.5) and Proposition 2.1. Hence, we may define

$$
\left\langle\left. F \cdot \nu\right|_{\partial \Omega}, \psi\right\rangle=\lim _{\varepsilon \rightarrow 0} l^{\varepsilon}(\psi), \quad \forall \psi \in \operatorname{Lip}(\gamma, \partial \Omega) .
$$

Now, for any $\phi \in \operatorname{Lip}\left(\gamma, \mathbb{R}^{N}\right)$, we let $\varepsilon \rightarrow 0$ in the Gauss-Green formula:

$$
\int_{\partial \Omega} F^{\varepsilon} \cdot \nu \phi d \mathcal{H}^{N-1}=\int_{\Omega} \phi \operatorname{div} F^{\varepsilon}+\int_{\Omega} \nabla \phi \cdot F^{\varepsilon}
$$

and obtain the identity (3.1).

For the general case that $F \in \mathcal{D M}^{\text {ext }}(\Omega)$ without the assumption (3.5), we consider a Lipschitz deformation of $\partial \Omega, \Psi: \partial \Omega \times[0,1] \rightarrow \bar{\Omega}$. Let $s \in(0,1)$ be such that

$$
|F|\left(\partial \Omega_{s}\right)=|\operatorname{div} F|\left(\partial \Omega_{s}\right)=0 .
$$

Since we have $\mathcal{H}^{N-1}\left(\partial \Omega_{s}\right)<+\infty$, Federer's extension of the Gauss-Green formula (see [13]) holds for $\phi F^{\varepsilon}$ over $\Omega_{s}$. Thus, we know from the previous analysis that $\left.F \cdot \nu\right|_{\partial \Omega_{s}}$ is defined as a continuous linear functional over $\operatorname{Lip}\left(\gamma, \partial \Omega_{s}\right)$, whose norm is bounded, independent of $s \in(0,1)$. Now, for $\psi \in \operatorname{Lip}(\gamma, \partial \Omega)$, we have

$$
\left\langle\left. F \cdot \nu\right|_{\partial \Omega_{s}},\left(\mathcal{E}(\psi) \mid \partial \Omega_{s}\right)\right\rangle=\int_{\Omega_{s}} \mathcal{E}(\psi) \operatorname{div} F+\int_{\Omega_{s}} \nabla \mathcal{E}(\psi) \cdot F .
$$

Again, the right-hand side of (3.7) defines a uniformly bounded family of continuous linear functionals $l^{s}$ over $\operatorname{Lip}(\gamma, \partial \Omega)$, for $s \in(0,1)-\mathcal{T}$, where $\mathcal{T}$ is defined in Lemma 3.1. Furthermore, $\lim _{s \rightarrow 0} l^{s}(\psi)$ exists for any $\psi \in \operatorname{Lip}(\gamma, \partial \Omega)$, as a consequence of the Dominated Convergence Theorem applied to both integrals on the right-hand side of (3.7). Hence, we may define

$$
\left\langle\left. F \cdot \nu\right|_{\partial \Omega}, \psi\right\rangle=\lim _{s \rightarrow 0} l^{s}(\psi),
$$

which is then a continuous linear functional over $\operatorname{Lip}(\gamma, \partial \Omega)$. Finally, for any $\phi \in$ $\operatorname{Lip}\left(\gamma, \mathbb{R}^{N}\right)$, we obtain (3.1) by taking the limit as $s \rightarrow 0$ in the formula:

$$
\left\langle\left. F \cdot \nu\right|_{\partial \Omega_{s}},\left(\phi \mid \partial \Omega_{s}\right)\right\rangle=\int_{\Omega_{s}} \phi \operatorname{div} F+\int_{\Omega_{s}} \nabla \phi \cdot F
$$

observing that

$$
\begin{aligned}
\mid\left\langle\left. F \cdot \nu\right|_{\partial \Omega_{s}},\right. & \left.\left(\phi \mid \partial \Omega_{s}\right)-\left(\mathcal{E}(\phi \mid \partial \Omega) \mid \partial \Omega_{s}\right)\right\rangle \mid \\
& \leq c\left\|\left(\phi \mid \partial \Omega_{s}\right)-\left(\mathcal{E}(\phi \mid \partial \Omega) \mid \partial \Omega_{s}\right)\right\|_{\operatorname{Lip}\left(\gamma^{\prime}, \partial \Omega_{s}\right)} \\
& \leq c\|\phi-\mathcal{E}(\phi \mid \partial \Omega)\|_{\operatorname{Lip}\left(\gamma^{\prime}, \Psi(\partial \Omega \times[0, s])\right)} \rightarrow 0, \quad \text { as } s \rightarrow 0,
\end{aligned}
$$

for $1<\gamma^{\prime}<\gamma$, as a consequence of Proposition 2.2.

Step 2. We now consider the more regular case that $F \in \mathcal{D M}^{p}(\Omega), 1 \leq p<\infty$. Let $F^{\varepsilon}$ be as above. Again, for any $\phi \in \operatorname{Lip}\left(\mathbb{R}^{N}\right)$, we have

$$
\int_{\partial \Omega_{s}} \phi F^{\varepsilon} \cdot \nu d \mathcal{H}^{N-1}=\int_{\Omega_{s}} \phi \operatorname{div} F^{\varepsilon} d x+\int_{\Omega_{s}} \nabla \phi \cdot F^{\varepsilon} d x
$$


Now we integrate (3.8) in $s \in(0, \delta), 0<\delta<1$, and use the coarea formula (see, e.g. $[12,13])$ in the left-hand side to obtain

$$
-\int_{\Psi(\Omega \times(0, \delta))} \phi F^{\varepsilon} \cdot \nabla h d x=\int_{0}^{\delta}\left\{\int_{\Omega_{s}} \phi \operatorname{div} F^{\varepsilon} d x\right\} d s+\int_{0}^{\delta}\left\{\int_{\Omega_{s}} \nabla \phi \cdot F^{\varepsilon} d x\right\} d s .
$$

Let $\varepsilon \rightarrow 0$. Observing that, by Proposition 2.1, the integrand of the first integral converges for a.e. $s \in(0, \delta)$ to the corresponding integral for $F$, we obtain

$$
-\int_{\Psi(\Omega \times(0, \delta))} \phi F \cdot \nabla h d x=\int_{0}^{\delta}\left\{\int_{\Omega_{s}} \phi \operatorname{div} F\right\} d s+\int_{0}^{\delta}\left\{\int_{\Omega_{s}} \nabla \phi \cdot F d x\right\} d s .
$$

We then divide (3.10) by $\delta$, let $\delta \rightarrow 0$, and observe that both terms in the righthand side converge to the corresponding integrals inside the brackets over $\Omega$, by the dominated convergence theorem. Hence, the left-hand side also converges, which yields

$$
-\lim _{\delta \rightarrow 0} \frac{1}{\delta} \int_{\Psi(\Omega \times(0, \delta))} \phi F \cdot \nabla h d x=\int_{\Omega} \phi \operatorname{div} F+\int_{\Omega} \nabla \phi \cdot F d x .
$$

Now, for $\psi \in \operatorname{Lip}(\partial \Omega)$, let $\mathcal{E}(\psi) \in \operatorname{Lip}\left(\mathbb{R}^{N}\right)$ be a Lipschitz extension of $\psi$ preserving the norm $\|\cdot\|_{\text {Lip }}:=\|\cdot\|_{\infty}+\operatorname{Lip}(\cdot)$ (see, e.g., $\left.[12,13]\right)$. We then define

$$
\left\langle\left. F \cdot \nu\right|_{\partial \Omega}, \psi\right\rangle=-\lim _{s \rightarrow 0} \frac{1}{s} \int_{\Psi(\partial \Omega \times(0, s))} \mathcal{E}(\psi) \nabla h \cdot F d x .
$$

Because the right-hand side of (3.11) does not depend on the particular deformation $\Psi$ for $\partial \Omega$, we see that the normal trace defined by (3.12) is also independent of the deformation.

We still have to prove that the normal trace as defined by (3.12) also does not depend on the specific Lipschitz extension $\mathcal{E}(\psi)$ of $\psi$. This will be accomplished if we prove that the right-hand side of (3.11) vanishes for $\left.\phi\right|_{\partial \Omega} \equiv 0$. Denote it by $[F, \phi]_{\partial \Omega}$, that is,

$$
[F, \phi]_{\partial \Omega}:=\langle\operatorname{div} F, \phi\rangle_{\Omega}+\langle F, \nabla \phi\rangle_{\Omega} .
$$

We claim that $[F, \phi]_{\partial \Omega}=0$ if $\left.\phi\right|_{\partial \Omega} \equiv 0$. In fact, we may approximate such $\phi$ by a sequence $\phi^{j} \in C_{0}^{\infty}(\Omega)$, with $\left\|\phi^{j}\right\|_{\infty} \leq\|\phi\|_{\infty}$, such that $\phi^{j} \rightarrow \phi$ locally uniformly in $\Omega$ and $\nabla \phi^{j} \rightarrow \nabla \phi$ in $L^{q}(\Omega)^{N}$, with $\frac{1}{p}+\frac{1}{q}=1$. Hence, $[F, \phi]_{\partial \Omega}=\lim _{j \rightarrow \infty}\left[F, \phi^{j}\right]=$ 0 , as asserted. In particular, for $1 \leq p \leq \infty$, the values of the normal trace, $\left\langle\left. F \cdot \nu\right|_{\partial \Omega},\left.\phi\right|_{\partial \Omega}\right\rangle$, depend only on the values of $\phi$ over $\partial \Omega$.

Step 3. The fact that formula (3.2) also holds if $F \in \mathcal{D} \mathcal{M}^{e x t}(\Omega)$, for any $\psi \in$ $\operatorname{Lip}(\gamma, \partial \Omega), \gamma>1$, provided that the set of non-Lebesgue points of $\nabla h(x)$ on $\Psi(\partial \Omega \times(0,1))$ has $|F|$-measure zero, is clear from the above proof for $\mathcal{D M}^{p}$-fields, $1 \leq p \leq \infty$.

Step 4. As for the last assertion, we recall a well-known result of Gagliardo [14] which indicates, in particular, that, if $\partial \Omega$ is Lipschitz (that is, satisfies (i) of Definition 3.1) and $\psi \in W^{1-1 / p, p}(\partial \Omega)$, then it can be extended into $\Omega$ to a function $\mathcal{E}(\psi) \in W^{1, p}(\Omega)$, and

$$
\|\mathcal{E}(\psi)\|_{W^{1, p}(\Omega)} \leq c\|\psi\|_{W^{1-1 / p, p}(\partial \Omega)},
$$

for some positive constant $c$ independent of $\psi$. ¿From the definition of $\mathcal{E}(\psi)$ given in [14], it is easy to verify that, when $\psi \in C(\partial \Omega), \mathcal{E}(\psi) \in C(\Omega)$ and $\|\mathcal{E}(\psi)\|_{L^{\infty}(\Omega)} \leq$ $\|\psi\|_{L^{\infty}(\partial \Omega)}$. Hence, using these facts and (3.11), we easily deduce the last assertion. 
Remark 3.1. When $F \in \mathcal{D} \mathcal{M}^{\infty}(D)$, the normal trace $\left.F \cdot \nu\right|_{\partial \Omega}$ is in fact in $L^{\infty}\left(\partial \Omega, \mathcal{H}^{N-1}\right)$ as the weak-star limit of $\left(F \cdot \nu_{s}\right) \circ \Psi_{s}$ in $L^{\infty}\left(\partial \Omega, \mathcal{H}^{N-1}\right)$ for any Lipschitz deformation $\Psi_{s}$ :

$$
\left.F \cdot \nu\right|_{\partial \Omega}=w^{*}-\lim \left(F \cdot \nu_{s}\right) \circ \Psi_{s}, \quad L^{\infty}\left(\partial \Omega, \mathcal{H}^{N-1}\right),
$$

which is independent of $\Psi_{s}$; and the weak-star topology for this limit is optimal to define $\left.F \cdot \nu\right|_{\partial \Omega}$ in general (see Chen-Frid [5]). However, for $F \in \mathcal{D M}^{*}(D)$, the normal traces $\left.F \cdot \nu\right|_{\partial \Omega}$ may no longer be functions in general. This can be seen in Example 1.1 for $F \in \mathcal{D} \mathcal{M}_{l o c}^{1}\left(\mathbb{R}^{2}\right)$ with $\Omega=(0,1) \times(0,1)$, for which

$$
\left.F \cdot \nu\right|_{\partial \Omega}=\frac{\pi}{2} \delta_{(0,0)}-\left.d \mathcal{H}^{1}\right|_{\partial \Omega},
$$

where $\mathcal{H}^{1}$ is the one-dimensional Hausdorff measure on $\partial \Omega$.

Remark 3.2. As mentioned in the proof of Theorem 3.1, if $F \in \mathcal{D} \mathcal{M}^{p}(\Omega)$, for $1 \leq p \leq \infty$, the values of the normal trace, $\left\langle\left. F \cdot \nu\right|_{\partial \Omega}, \phi \mid \partial \Omega\right\rangle$, depend only on the values of $\phi$ over $\partial \Omega$. In contrast, for $F \in \mathcal{D} \mathcal{M}^{e x t}(\Omega)$, the values of $\left\langle\left. F \cdot \nu\right|_{\partial \Omega}, \phi \mid \partial \Omega\right\rangle$ also depend, in principle, on the values of the first derivatives of $\phi$ over $\partial \Omega$, since $\phi \mid \partial \Omega$ must be viewed as elements of $\operatorname{Lip}(\gamma, \partial \Omega)$, for some $\gamma>1$.

Finally, we establish the following useful product rule.

Theorem 3.2 (Product Rule). Let $F=\left(F_{1}, \cdots, F_{N}\right) \in \mathcal{D M}^{*}(D)$. Let $g \in C(D)$ be such that $\partial_{x_{j}} g(x)$ is $\left|F_{j}\right|$-integrable, for each $j=1, \cdots, N$, and the set of nonLebesgue points of $\partial_{x_{j}} g(x)$ has $\left|F_{j}\right|$-measure zero. Then $g F \in \mathcal{D M}^{*}(D)$ and

$$
\operatorname{div}(g F)=g \operatorname{div} F+\nabla g \cdot F .
$$

Proof. For any $\phi \in C_{0}^{1}(D)$, we have

$$
\langle\operatorname{div}(g F), \phi\rangle=-\langle F, g \nabla \phi\rangle=-\langle F, \nabla(g \phi)\rangle+\langle F, \phi \nabla g\rangle .
$$

Therefore, it suffices to show that

$$
\langle F, \nabla(g \phi)\rangle=-\langle\operatorname{div} F, g \phi\rangle .
$$

Set $g^{\varepsilon}=g * \omega^{\varepsilon}$. We have

$$
\begin{aligned}
-\left\langle\operatorname{div} F, g^{\varepsilon} \phi\right\rangle & =\left\langle F, \nabla\left(g^{\varepsilon} \phi\right)\right\rangle=\sum_{j=1}^{N}\left\langle F_{j}, \partial_{x_{j}}\left(g^{\varepsilon} \phi\right)\right\rangle \\
& =\sum_{j=1}^{N}\left\{\left\langle F_{j}, \phi \partial_{x_{j}} g^{\varepsilon}\right\rangle+\left\langle F_{j}, g^{\varepsilon} \partial_{x_{j}} \phi\right\rangle\right\} .
\end{aligned}
$$

Let $\varepsilon \rightarrow 0$. Then the right-hand side converges to

$$
\sum_{j=1}^{N}\left\{\left\langle F_{j}, \phi \partial_{x_{j}} g\right\rangle+\left\langle F_{j}, g \partial_{x_{j}} \phi\right\rangle\right\}=\langle F, \nabla(g \phi)\rangle,
$$

by the assumption on the set of non-Lebesgue points of $\partial_{x_{j}} g$, while the left-hand side of (3.17) converges to $-\langle\operatorname{div} F, g \phi\rangle$ by the Dominated Convergence Theorem, which implies (3.16). Then (3.14) follows. 
Remark 3.3. The continuity assumption of $g(x)$ in Theorem 3.3 can be relaxed. In particular, when $F \in \mathcal{D} \mathcal{M}^{\infty}(D)$, it requires only that $g \in B V(D ; \mathbb{R})$ to have $g F \in \mathcal{D M}^{\infty}(D)$.

Remark 3.4. The results in this section for the extended vector fields over $\mathbb{R}^{N}$ extend to a general context over Riemannian manifolds. In particular, if $a_{i j}(x)$ are smooth functions on the open set $D \subset \mathbb{R}^{N}$ and the $N \times N$ matrix $\left(a_{i j}\right)(x)$ is symmetric, positive definite, the results can easily be generalized to the extended vector fields $F=\left(F_{1}, \cdots, F_{N}\right)$ in $L^{p}\left(D ; \mathbb{R}^{N}\right), 1 \leq p \leq \infty$, or $M\left(D ; \mathbb{R}^{N}\right)$, satisfying the condition:

$$
\sum_{i, j} a_{i j} \partial_{i} F_{j} \in \mathcal{M}(D)
$$

This is clear from the fact that no specific property of the Euclidean metric has been used in our analysis.

\section{Applications to the Euler Equations for Gas Dynamics}

In this section, as a direct application of the theory developed in Section 3, we establish the uniqueness and stability of Riemann solutions that may contain vacuum for the Euler equations for gas dynamics in Lagrangian coordinates. We will address more applications of the theory in forthcoming papers.

Denote $\mathbb{R}_{+}^{2}=(0, \infty) \times \mathbb{R}$ and $\overline{\mathbb{R}_{+}^{2}}=[0, \infty) \times \mathbb{R}$. We consider $\tau \in \mathcal{M}^{+}\left(\mathbb{R}_{+}^{2}\right)$ satisfying $\tau \geq c \mathcal{L}^{2}$ for some $c>0$, where $\mathcal{L}^{k}$ is the $k$-dimensional Lebesgue measure. Let $v \in L^{\infty}\left(\mathbb{R}_{+}^{2}\right)$ and $\tau_{0} \in \mathcal{M}^{+}(\mathbb{R})$ with $\tau_{0} \geq c \mathcal{L}^{1}$. We assume that $\tau, v$, and $\tau_{0}$ satisfy

$$
\iint_{\mathbb{R}_{+}^{2}}\left(\phi_{t} \tau-v \phi_{x} d t d x\right)+\int_{\mathbb{R}} \phi(0, x) \tau_{0}(x)=0,
$$

for any $\phi \in C_{0}^{1}\left(\mathbb{R}^{2}\right)$.

Definition 4.1. Let $\tau$ and $\tau_{0}$ be as above. We say that a function $\phi(t, x)$ defined on $\overline{\mathbb{R}_{+}^{2}}$ is a $\tau$-test function if it satisfies the following:

(1) $\operatorname{spt}(\phi)$ is a compact subset of $\overline{\mathbb{R}_{+}^{2}}$ and $\phi$ is continuous on $\mathbb{R}_{+}^{2}$;

(2) $\phi_{t}$ and $\phi_{x}$ are $\tau$-measurable; and $\phi_{t}$ is $\tau$-integrable over $\mathbb{R}_{+}^{2}$, that is, the integrals $\iint_{\mathbb{R}_{+}^{2}}\left(\phi_{t}\right)_{ \pm} \tau$ exist and at least one of them is finite;

(3) $\lim _{\substack{t \rightarrow 0 \\ x \rightarrow a}} \phi(t, x)=\phi(0, a)$ for $\tau_{0}$-a.e. $a \in \mathbb{R}$.

Theorem 4.1. Let $\tau, v$, and $\tau_{0}$ be as above. Then

(1) the nonnegative measure $\tau$ admits a slicing of the form $\tau=d t \otimes \mu_{t}(x)$ with $\mu_{t} \in \mathcal{M}^{+}(\mathbb{R})$ for $\mathcal{L}^{1}$-a.e. $t>0$. More precisely, for all $\phi \in C_{0}\left(\mathbb{R}_{+}^{2}\right)$,

$$
\iint \phi(t, x) \tau=\int\left(\int \phi(t, x) \mu_{t}(x)\right) d t .
$$

(2) the points $(t, x) \in \mathbb{R}_{+}^{2}$ such that $\mu_{t}(x)>0$, with the exception of a set of $\mathcal{H}^{1}$-measure zero, form a countable union of vertical line segments, called vacuum lines. In particular, $\tau(l)=0$ for any non-vertical straight line segment $l$.

(3) the identity (4.1) holds for any $\tau$-test function $\phi(t, x)$.

The proof of Theorem 4.1 is given in Section 5. As a corollary, we have 
Corollary 4.1. Let $\tau, v$, and $\tau_{0}$ be as above. Let $\bar{p}(t, x)$ be a nonnegative function over $\overline{\mathbb{R}_{+}^{2}}$, continuous on $\mathbb{R}_{+}^{2}$, such that $\phi \bar{p}$ is a $\tau$-test function for any $\phi \in C_{0}^{1}\left(\mathbb{R}^{2}\right)$, $\bar{p}_{t} \leq 0$, $\tau$-a.e., and $\bar{p}_{x} \in L_{l o c}^{1}\left(\mathbb{R}_{+}^{2}\right)$. Then, for any nonnegative function $\zeta \in C_{0}^{1}(\mathbb{R})$,

$$
\limsup _{t \rightarrow 0+} \int \zeta(x) \bar{p}(t, x) \mu_{t}(x) \leq \int \zeta(x) \bar{p}(0, x) \tau_{0}(x) .
$$

Proof. First we have from Theorem 4.1 that (4.1) holds for $\phi=\bar{p} \psi$ with $\psi \in$ $C_{0}^{1}\left(\mathbb{R}^{2}\right)$. Then we choose $\psi=\psi^{\varepsilon}(t, x):=\sigma^{\varepsilon}\left(t-t_{0}\right) \zeta(x)$ with $\zeta(x) \geq 0, t_{0}>0$, and

$$
\begin{aligned}
& 0 \leq \sigma^{\varepsilon} \in C_{0}^{1}(\mathbb{R}), \\
& \sigma^{\varepsilon}\left(t-t_{0}\right) \rightarrow \chi_{\left(-\infty, t_{0}\right)}(t), \\
& \delta^{\varepsilon}\left(t-t_{0}\right)=-\frac{d}{d t} \sigma^{\varepsilon}\left(t-t_{0}\right) \rightarrow \delta_{t_{0}}, \quad \text { as } \varepsilon \rightarrow 0,
\end{aligned}
$$

where $\delta_{t_{0}}$ is the Dirac measure concentrated at $t_{0}$, and the convergences are in $L^{1}(\mathbb{R})$ and $\mathcal{M}(\mathbb{R})$, respectively. We obtain from (4.1) that

$$
\begin{aligned}
0 & =\iint_{t>0}\left(\left(\psi^{\varepsilon} \bar{p}\right)_{t} \mu_{t} d t-\left(\psi^{\varepsilon} \bar{p}\right)_{x} v d t d x\right)+\int\left(\psi^{\varepsilon} \bar{p}_{0}\right) \tau_{0} \\
& =-\int \delta^{\varepsilon}\left(\int \zeta \bar{p} \mu_{t}\right) d t+\iint \bar{p}_{t} \psi^{\varepsilon} \mu_{t} d t-\iint\left(\psi^{\varepsilon} \bar{p}\right)_{x} v d t d x+\int\left(\psi^{\varepsilon} \bar{p}_{0}\right) \tau_{0} .
\end{aligned}
$$

Now, using that $\bar{p}_{t} \leq 0, \tau$-a.e., we obtain

$$
-\int \delta^{\varepsilon}\left(\int \zeta \bar{p} \mu_{t}\right) d t+C_{0} \iint_{\substack{0 \leq t \leq t_{0} \\|x| \leq|\operatorname{spt}(\zeta)|}}\left(\left|\bar{p}_{x}\right|+\left|\zeta^{\prime}\right|\right) d t d x+\int\left(\psi^{\varepsilon} \bar{p}_{0}\right) \tau_{0} \geq 0 .
$$

Assuming that $t_{0}$ is a Lebesgue point of $g(t)=\int \zeta \bar{p} \mu_{t}$ and letting $\varepsilon \rightarrow 0$ yield

$$
\int \zeta \bar{p} \mu_{t_{0}} \leq C \iint_{\substack{0 \leq t \leq t_{0} \\|x| \leq|\operatorname{spt}(\zeta)|}}\left(\left|\bar{p}_{x}\right|+\left|\zeta^{\prime}\right|\right) d t d x+\int \zeta \bar{p}_{0} \tau_{0} .
$$

Now, taking the limsup as $t_{0} \rightarrow 0$ in both sides of (4.3), we finally arrive at (4.2).

We now consider the solutions of the Euler equations (1.1)-(1.3) for gas dynamics in the sense of distributions such that $\tau$ is a nonnegative Radon measure, with $\tau \geq c \mathcal{L}^{2}$ for some $c>0$, and $v(t, x)$ and $S(t, x)$ are bounded $\tau$-measurable functions, along with our understanding that the constitutive relations (1.4) for $(\tau, p, e, \theta, S)(t, x)$ hold $\mathcal{L}^{2}$-almost everywhere out of the vacuum lines, in the set where $\tau$ is absolutely continuous with respect to $\mathcal{L}^{2}$, and both $p(t, x)$ and $e(t, x)$ are defined as zero on the remaining set with measure zero in $\mathbb{R}_{+}^{2}$, including the vacuum lines.

We consider the Cauchy problem for (1.1)-(1.3):

$$
\left.(\tau, v, S)\right|_{t=0}=\left(\tau_{0}, v_{0}, S_{0}\right)(x),
$$

where $\tau_{0}(x)$ is a nonnegative Radon measure over $\mathbb{R}, \tau_{0} \geq c \mathcal{L}^{1}$ for some $c>0, v_{0}(x)$ and $S_{0}(x)$ are bounded $\tau_{0}$-measurable functions, and $e_{0}(x)=e\left(\tau_{0}(x), S_{0}(x)\right)$ a.e. out of the countable points $\left\{x_{k}\right\}$ such that $\tau_{0}\left(x_{k}\right)>0$, the initial vacuum set. 
Set $\Pi_{T}=(0, T) \times \mathbb{R}$ and $\Pi_{T}^{*}=(-\infty, T) \times \mathbb{R}$ for $T>0$. Let $D$ and $F$ be functions or measures over $\Pi_{T}$. Let $D_{0}$ be a function or a measure over $\mathbb{R}$. By weak formulation on $\Pi_{T}$ for the Cauchy problem:

$$
\begin{aligned}
& D_{t}+F_{x}=0, \\
& \left.D\right|_{t=0}=D_{0},
\end{aligned}
$$

we mean that, for a suitable set of test functions $\phi(t, x)$ defined on $\Pi_{T}^{*}$,

$$
\iint_{\Pi_{T}}\left(\phi_{t} D+\phi_{x} F\right)+\int_{\mathbb{R}} \phi(0, x) D_{0}=0
$$

Analogously, if the identity " $="$ in (4.5) is replaced by " $\geq$ " or " $\leq$ ", the weak formulation of the corresponding problem (4.5) and (4.6) is (4.7) with " =" replaced by " $\leq$ " or " $\geq$ ", respectively, for a suitable set of nonnegative test functions defined on $\Pi_{T}^{*}$.

Denote $W=(\tau, v, S), f(W)=(-v, p(\tau, S), 0), \eta(W)=e(\tau, S)+\frac{v^{2}}{2}, q(W)=$ $v p(\tau, S)$, and

$$
\begin{aligned}
& \alpha(W, \bar{W})=\eta(W)-\eta(\bar{W})-\nabla \eta(\bar{W}) \cdot(W-\bar{W}), \\
& \beta(W, \bar{W})=q(W)-q(\bar{W})-\nabla \eta(\bar{W}) \cdot(f(W)-f(\bar{W})) .
\end{aligned}
$$

Observe that $\nabla \eta(\bar{W})=(-\bar{p},-\bar{v}, \bar{\theta})$.

Definition 4.2. We say that $W(t, x)$ is a distributional entropy solution of (1.1)(1.3), and (4.4) in $\Pi_{T}$ if $\tau$ is a Radon measure on $\Pi_{T}$ with $\tau \geq c \mathcal{L}^{2}$ for some $c>0, v$ and $S$ are bounded $\tau$-measurable functions such that the weak formulation of (1.1)-(1.3), (1.7), and (4.4) is satisfied for all test functions in $C_{0}^{1}\left(\Pi_{T}^{*}\right)$, and $S(t, \cdot) \rightarrow S_{0}(\cdot)$, as $t \rightarrow 0$, in the weak-star topology of $L^{\infty}(\mathbb{R})$.

Observe that the weak formulation implies that $\mu_{t} \rightarrow \tau_{0}$ in $\mathcal{M}(\mathbb{R})$, and $v(t, \cdot) \rightarrow$ $v_{0}(\cdot)$, and $E(t, \cdot) \rightarrow E_{0}(\cdot)$ in the weak-star topology of $L^{\infty}(\mathbb{R})$, as $t \rightarrow 0$, where $E=e+v^{2} / 2$. We also remark that these convergences can be strengthened to the convergences in $L_{l o c}^{1}(\mathbb{R})$ in the case that $\tau$ is a bounded measurable function, as an easy consequence of the $\mathcal{D} \mathcal{M}^{\infty}$ theory (cf. [5]).

As shown by Wagner [25], by means of the transformation from Eulerian to Lagrangian coordinates, bounded measurable entropy solutions of the Euler equations in Eulerian coordinates transform into distributional entropy solutions of (1.1)-(1.3) and (4.4), satisfying the additional restriction that the weak formulation of (1.2), (1.3), and (1.7) holds for test functions with compact support in $\Pi_{T}$ such that $\phi_{t}=g, \phi_{x}=h \tau$, where $g, h \in L^{\infty}\left(\Pi_{T}, \tau\right)$. It is also shown through an example in [25] that distributional entropy solutions without the additional restriction may have no physical meaning.

Now we consider the Riemann solution $\bar{W}(t, x)$ associated to the Riemann problem for (1.1)-(1.3) with initial condition

$$
\bar{W}_{0}(x)= \begin{cases}W_{L}, & x<0, \\ W_{R}, & x>0,\end{cases}
$$

where $W_{L}$ and $W_{R}$ are two constant states in the physical domain $\{W=(\tau, v, S)$ : $\tau>0\}$. First, we address the case that $\bar{W}(t, x)$ is a bounded self-similar entropy 
solution of (1.1)-(1.3) which consists of at most two rarefaction waves, one corresponding to the first characteristic family and another corresponding to the third one, and possibly one contact discontinuity on the line $x=0$. Then, $\bar{W}(t, x)$ has the following general form:

$$
\bar{W}(x, t)= \begin{cases}W_{L}, & x / t<\xi_{1}, \\ R_{1}(x / t), & \xi_{1} \leq x / t<\xi_{2}, \\ W_{M}, & \xi_{2} \leq x / t<0, \\ W_{N}, & 0<x / t<\xi_{3}, \\ R_{3}(x / t), & \xi_{3} \leq x / t<\xi_{4}, \\ W_{R}, & x / t \geq \xi_{4} .\end{cases}
$$

In what follows we use the notation $W^{p}=(\tau, S), \bar{W}^{p}=(\bar{\tau}, \bar{S})$.

Theorem 4.2. Let $\bar{W}(t, x)$ be the Riemann solution (4.9), and let $W(t, x)$ be any distributional entropy solution of (1.1)-(1.3) and (4.4) with $W_{0} \in L^{\infty}\left(\mathbb{R} ; \mathbb{R}^{3}\right)$. Then there exist positive constants $C$ and $K_{0}$, and a function $\omega \in L^{\infty}\left(\Pi_{T}\right)$, positive a.e. in $\Pi_{T}$, such that, for any $X>0$ and a.e. $t>0$,

$$
\begin{aligned}
\int_{|x| \leq X} & \left(|v(t, x)-\bar{v}(t, x)|^{2}+\left|W_{a . c .}^{p}(t, x)-\bar{W}^{p}(t, x)\right|^{2} \omega(t, x)\right) d x \\
\leq C & \int_{|x| \leq X+K_{0} t}\left(\left|v_{0}(x)-\bar{v}_{0}(x)\right|^{2}+\left|W_{0}^{p}(x)-\bar{W}_{0}^{p}(x)\right|^{2} \omega(0, x)\right) d x .
\end{aligned}
$$

Proof. We divide the proof into four steps.

Step 1. Consider the measure

$$
\varpi:=\alpha(W, \bar{W})_{t}+\beta(W, \bar{W})_{x} .
$$

Given any $X>0$ and $t>0$, let $t_{0} \in(0, t)$ and

$$
\Omega_{t_{0}, t}=\left\{(\sigma, x):|x|<X+K_{0}(t-\sigma), t_{0}<\sigma<t\right\},
$$

for $K_{0}>0$ to be suitably chosen later.

First, by the Gauss-Green formula (Theorem 3.1), we have

$$
\varpi\left(\Omega_{t_{0}, t}\right)=\left\langle\left.(\alpha, \beta) \cdot \nu\right|_{\partial \Omega_{t_{0}, t}}, 1\right\rangle
$$

Now, let $\zeta_{i}, i=1, \cdots, 4$, be nonnegative functions in $C_{0}^{\infty}\left(\mathbb{R}^{2}\right)$ such that

$$
\begin{aligned}
& \sum_{i=1}^{4} \zeta_{i}=1, \quad \text { over } \partial \Omega_{t_{0}, t}, \\
& \zeta_{1}=1, \quad \text { over }\left\{(0, x):|x|<X+K_{0}\left(t-t_{0}\right)\right\}, \\
& \zeta_{2}=1, \quad \text { over }\{(t, x):|x|<X\}, \\
& \left(\operatorname{spt}\left(\zeta_{3}\right) \cup \operatorname{spt}\left(\zeta_{4}\right)\right) \cap\left((\{t\} \times \mathbb{R}) \cup\left(\left\{t_{0}\right\} \times \mathbb{R}\right)\right)=\emptyset .
\end{aligned}
$$

We choose $\zeta_{3}$ and $\zeta_{4}$ so that spt $\left(\zeta_{3}\right)$ intersects the left lateral side of $\Omega_{t_{0}, t}$ but not the right, and spt $\left(\zeta_{4}\right)$ intersects its right lateral side but not the left. We have

$$
\varpi\left(\Omega_{t_{0}, t}\right)=\left\langle\left.(\alpha, \beta) \cdot \nu\right|_{\partial \Omega_{t_{0}, t}}, \zeta_{1}+\zeta_{2}+\zeta_{3}+\zeta_{4}\right\rangle .
$$

In what follows, we will use the notations:

$$
\hat{g}=\int_{0}^{1}(1-\sigma) g(\sigma \tau+(1-\sigma) \bar{\tau}, \sigma S+(1-\sigma) \bar{S}) d \sigma
$$


for any function $g=g(\tau, S)$, and

$$
\tilde{g}=\int_{0}^{1} g(\sigma \tau+(1-\sigma) \bar{\tau}, \sigma S+(1-\sigma) \bar{S}) d \sigma .
$$

Step 2. We now prove the following five estimates.

1. $\left\langle\left.(\alpha, \beta) \cdot \nu\right|_{\partial \Omega_{t_{0}, t}}, \zeta_{3}\right\rangle \geq 0$ and $\left\langle\left.(\alpha, \beta) \cdot \nu\right|_{\partial \Omega_{t_{0}, t}}, \zeta_{4}\right\rangle \geq 0$.

Indeed, let $z_{*}=\left(t_{*}, x_{*}\right)$ be the center of $\Omega_{t_{0}, t}$. We consider the following deformation of $\partial \Omega_{t_{0}, t}$ :

$$
\Psi(z, s):=z+\varepsilon s\left(z_{*}-z\right), \quad z \in \partial \Omega_{t_{0}, t}, s \in[0,1],
$$

where $\varepsilon$ is chosen so small that

$\left(\operatorname{spt}\left(\zeta_{3}\right) \cup \operatorname{spt}\left(\zeta_{4}\right)\right) \cap\left(\left\{\left(t-\varepsilon s\left(t-t_{*}\right), x\right): x \in \mathbb{R}\right\} \cup\left\{\left(t_{0}+\varepsilon s\left(t_{*}-t_{0}\right), x\right): x \in \mathbb{R}\right\}\right)=\emptyset$, for $s \in[0,1]$. Then

$$
\left\langle\left.(\alpha, \beta) \cdot \nu\right|_{\partial \Omega_{t_{0}, t}}, \zeta_{3}\right\rangle=\lim _{s \rightarrow 0} \frac{1}{s} \iint_{\Psi\left(\partial \Omega_{t_{0}, t} \times(0, s)\right)}\left(K_{0} \alpha-\beta\right)|\nabla h| \zeta_{3} d \sigma d x \geq 0,
$$

by choosing $K_{0}$ such that $K_{0} \alpha \geq \beta$, which is possible by what follows.

2. Given $\delta>0$ and bounded sets $B_{1} \subset V_{\delta}:=\{(\tau, v, S): \tau>\delta\}$ and $B_{2} \subset \mathbb{R}^{2}$, there exists a constant $K_{0}=K_{0}\left(\delta, B_{1}, B_{2}\right)>0$ such that $K_{0} \alpha(W, \bar{W}) \geq \beta(W, \bar{W})$, for any $\bar{W} \in B_{1}$ and $W \in V_{\delta}$ with $(v, S) \in B_{2}$.

In fact, we first have

$$
\begin{aligned}
& \alpha(W, \bar{W})=\frac{1}{2}(v-\bar{v})^{2}+\hat{e}_{\tau \tau}(\tau-\bar{\tau})^{2}+2 \hat{e}_{\tau S}(\tau-\bar{\tau})(S-\bar{S})+\hat{e}_{S S}(S-\bar{S})^{2}, \\
& \beta(W, \bar{W})=(v-\bar{v})(p-\bar{p}) .
\end{aligned}
$$

If $W(t, x)$ and $\bar{W}(t, x)$ belong to a bounded set $B$ in $V_{\delta}$, we can find $K_{0}$ depending only on $B$ such that $K_{0} \alpha \geq \beta$. Now, since $(\bar{\tau}, \bar{v}, \bar{S}) \in B_{1}$ and $(v, S) \in B_{2}$, it suffices to show that, for $\tau$ sufficiently large, we have $\beta(W, \bar{W}) \leq \alpha(W, \bar{W})$. Notice that

$$
\begin{aligned}
\beta & \leq \frac{1}{2}(v-\bar{v})^{2}+\frac{1}{2} \tilde{p}_{\tau}^{2}(\tau-\bar{\tau})^{2}+\tilde{p}_{\tau} \tilde{p}_{S}(\tau-\bar{\tau})(S-\bar{S})+\frac{1}{2} \tilde{p}_{S}^{2}(S-\bar{S})^{2} \\
& \leq \frac{1}{2}(v-\bar{v})^{2}+\tilde{p}_{\tau}^{2}(\tau-\bar{\tau})^{2}+\tilde{p}^{2}(S-\bar{S})^{2} .
\end{aligned}
$$

On the other hand,

$$
\alpha \geq \frac{1}{2}(v-\bar{v})^{2}+\frac{\hat{e}_{\tau \tau}}{2(K+1)}(\tau-\bar{\tau})^{2}+\frac{1}{2}\left(\hat{e}_{S S}-\frac{K+1}{K} \widehat{\left(\frac{e_{\tau S}^{2}}{e_{\tau \tau}}\right)}\right)(S-\bar{S})^{2},
$$

for any $K>0$. Now, $\tilde{p}_{\tau}^{2}$ decays faster than $\hat{e}_{\tau \tau}=-\hat{p}_{\tau}$ as $\tau \rightarrow \infty, \tilde{p}_{S}^{2}$ decays faster than $\hat{e}_{S S}$ as $\tau \rightarrow \infty$, and, for $K$ sufficiently large,

$$
\hat{e}_{S S}-\frac{K+1}{K} \widehat{\left(\frac{e_{\tau S}^{2}}{e_{\tau \tau}}\right)}>c \hat{e}_{S S}
$$

for some $c>0$ sufficiently small, since $\gamma>1$, as one can easily check from (1.4).

Hence, $\beta(W, \bar{W}) \leq \alpha(W, \bar{W})$ for $\tau$ larger than a certain $\tau_{*}$. Then the assertion follows.

3. Similarly, we have $\left\langle\left.(\alpha, \beta) \cdot \nu\right|_{\partial \Omega_{t_{0}, t}}, \zeta_{4}\right\rangle \geq 0$. 
4. As for $\zeta_{2}$, we have

$$
\begin{aligned}
& \left\langle\left.(\alpha, \beta) \cdot \nu\right|_{\partial \Omega_{t_{0}, t}}, \zeta_{2}\right\rangle=\lim _{s \rightarrow 0} \frac{1}{s} \iint_{\Psi\left(\partial \Omega_{t_{0}, t} \times(0, s)\right)}(\alpha, \beta) \cdot \nabla h \zeta_{2} d \sigma d x \\
& \geq \lim _{s \rightarrow 0} \frac{1}{\varepsilon\left(t-t_{*}\right) s} \int_{t-\varepsilon s\left(t-t_{*}\right)}^{t} d \sigma \int_{|x| \leq X-\varepsilon K_{1} \frac{T}{2}}\left\{\eta(W)-\eta(\bar{W})-\bar{v}(v-\bar{v})+\bar{p}\left(\tau_{\text {a.c. }}-\bar{\tau}\right)+\bar{\theta}(S-\bar{S})\right\} d x \\
& =\int_{\substack{|x| \leq X-\varepsilon K_{1} \frac{T}{2} \\
\sigma=t}}\left\{\eta\left(W_{\text {a.c. }}\right)-\eta(\bar{W})-\bar{v}(v-\bar{v})+\bar{p}\left(\tau_{\text {a.c. }}-\bar{\tau}\right)+\bar{\theta}(S-\bar{S})\right\} d x \\
& =\int_{\substack{|x| \leq X-\varepsilon K_{1} \frac{T}{2} \\
\sigma=t}}\left(\frac{1}{2}|v-\bar{v}|^{2}+Q\left(W_{\text {a.c. }}^{p}(t, x)-\bar{W}^{p}(t, x)\right)\right) d x
\end{aligned}
$$

if $t$ is a Lebesgue point of

$$
g(s)=\int_{|x| \leq X-\varepsilon K_{1} \frac{T}{2}} \alpha\left(W_{\text {a.c. }}, \bar{W}\right)(s, x) d x,
$$

where $Q$ is the quadratic form associated with the symmetric matrice

$$
A=\left[\begin{array}{ll}
\hat{e}_{\tau \tau} & \hat{e}_{\tau S} \\
\hat{e}_{\tau S} & \hat{e}_{S S}
\end{array}\right]
$$

5. For $\zeta_{1}$, we have

$$
\begin{aligned}
& \left\langle(\alpha, \beta) \cdot \nu \mid \partial \Omega_{t_{0}, t}, \zeta_{1}\right\rangle=\lim _{s \rightarrow 0} \frac{1}{s} \iint_{\Psi\left(\partial \Omega_{t_{0}, t} \times(0, s)\right)}(\alpha, \beta) \cdot \nu \zeta_{1}|\nabla h| d \sigma d x \\
& \geq-\lim _{s \rightarrow 0} \frac{1}{s} \int_{t_{0}}^{t_{0}+\varepsilon s\left(t_{*}-t_{0}\right)} \int_{|x| \leq X+K_{0}\left(t-t_{0}\right)} \alpha(W, \bar{W}) \frac{1}{\varepsilon\left(t_{*}-t_{0}\right)} d \sigma d x \\
& =-\int_{\substack{|x| \leq X+K_{0}\left(t-t_{0}\right) \\
\sigma=t_{0}}}\left\{\eta(W)-\eta(\bar{W})-\bar{v}(v-\bar{v})+\bar{p}\left(\mu_{t_{0}}-\bar{\tau}\right)-\bar{\theta}(S-\bar{S})\right\},
\end{aligned}
$$

where we have also used that $\mu_{\sigma} \rightarrow \mu_{t_{0}}$ as $\sigma \rightarrow t_{0}+0$, for a.e. $t_{0}>0$, with $\mu_{t}$ as in Theorem 4.1, and that $\bar{p}$ is continuous on $\left[t_{0}, t\right] \times \mathbb{R}$.

Step 3. On the other hand,

$$
\begin{aligned}
\varpi\left(\Omega_{t_{0}, t}\right)= & \sum_{i=1}^{4} \varpi\left(\tilde{l}_{i} \cap \Omega_{t_{0}, t}\right)+\varpi\left(l \cap \Omega_{t_{0}, t}\right)+\varpi\left(\Omega_{1} \cap \Omega_{t_{0}, t}\right) \\
& +\varpi\left(\Omega_{3} \cap \Omega_{t_{0}, t}\right)+\varpi\left(\Omega_{t_{0}, t}-\left(\cup_{i=1}^{4} \tilde{l}_{i} \cup l \cup \Omega_{1} \cup \Omega_{3}\right)\right),
\end{aligned}
$$

where $\Omega_{1}$ and $\Omega_{3}$ are the left and right rarefaction regions, $\tilde{l}_{i}, 1 \leq i \leq 4$, are the lines bounding the rarefaction regions $\Omega_{1}$ and $\Omega_{3}$, and $l$ is the line $\{x=0\}$ where $\bar{W}(t, x)$ has a contact discontinuity.

We first observe that, on $\Omega_{t_{0}, t}-\left(\cup_{i=1}^{4} \tilde{l}_{i} \cup l \cup \Omega_{1} \cup \Omega_{3}\right)$, the measure $\varpi$ reduces to $-\bar{\theta} \partial_{t} S$ which is nonpositive. Now, we have

$$
\varpi=-\operatorname{div}\left(F_{1}+F_{2}+F_{3}\right)
$$


where

$$
F_{1}=\bar{v}(v-\bar{v}, p-\bar{p}), \quad F_{2}=-\bar{p}(\tau-\bar{\tau}, \bar{v}-v), \quad F_{3}=\bar{\theta}(S-\bar{S}, 0),
$$

and $\operatorname{div}:=\operatorname{div}_{t, x}$. Applying the product rule (Theorem 3.2), we get

$$
\operatorname{div} F_{1}=\bar{v}_{t}(v-\bar{v})+\bar{v}_{x}(p-\bar{p}), \quad \operatorname{div} F_{2}=-\bar{p}_{t}(\tau-\bar{\tau})+\bar{p}_{x}(v-\bar{v}) .
$$

Hence,

$$
\operatorname{div} F_{1}\left(\tilde{l}_{j} \cap \Omega_{t_{0}, t}\right)=\operatorname{div} F_{1}\left(l \cap \Omega_{t_{0}, t}\right)=0, \quad j=1, \cdots, 4,
$$

since $\operatorname{div} F_{1}$ is absolutely continuous with respect to $\mathcal{L}^{2}$. Also,

$$
\operatorname{div} F_{3}\left(\tilde{l}_{j} \cap \Omega_{t_{0}, t}\right) \geq 0, \quad j=1, \cdots, 4 .
$$

On the other hand, since $F_{3} \in \mathcal{D} \mathcal{M}^{\infty}\left(\Omega_{t_{0}, t}\right)$ and $\left.\nu\right|_{l}=(0,1)$, we have

$$
\operatorname{div} F_{3}\left(l \cap \Omega_{t_{0}, t}\right)=\left[\left\langle\left. F_{3} \cdot \nu\right|_{l}, 1\right\rangle\right]=0,
$$

where the square-bracket denotes the difference between the normal traces from the right and the left, which make sense for $F_{3} \in \mathcal{D} \mathcal{M}^{\infty}$ because the normal traces of $\mathcal{D} \mathcal{M}^{\infty}$ fields are functions in $L^{\infty}$ over the boundaries.

Concerning $F_{2}$, we have

$$
\operatorname{div} F_{2}\left(\tilde{l}_{j} \cap \Omega_{t_{0}, t}\right)=0, \quad j=1, \cdots, 4,
$$

since $\bar{p}_{t}$ is $\tau$-integrable and $\tau\left(\tilde{l}_{j}\right)=0, j=1, \cdots, 4$. On the other hand, $\bar{p}_{t}$ vanishes on $l$ so that

$$
\operatorname{div} F_{2}\left(l \cap \Omega_{t, t_{0}}\right)=0 .
$$

Finally, using the product rule (Theorem 3.2), the fact that $W(t, x)$ and $\bar{W}(t, x)$ are distributional solutions of (1.1)-(1.3) and (4.4), and $\bar{S}(t, x)_{t}=0$, we obtain, for $j=1,3$,

$$
\begin{aligned}
\varpi\left(\Omega_{j} \cap \Omega_{t_{0}, t}\right) & =-\iint_{\Omega_{j} \cap \Omega_{t_{0}, t}}\left\{(\bar{v}(v-\bar{v}))_{t}+(\bar{v}(p-\bar{p}))_{x}-(\bar{p}(\tau-\bar{\tau}))_{t}+(\bar{p}(v-\bar{v}))_{x}+(\bar{\theta}(S-\bar{S}))_{t}\right\} \\
& =-\iint_{\Omega_{j} \cap \Omega_{t_{0}, t}}\left\{\bar{v}_{t}(v-\bar{v})+\bar{v}_{x}(p-\bar{p})-\bar{p}_{t}(\tau-\bar{\tau})+\bar{p}_{x}(v-\bar{v})+\bar{\theta}_{t}(S-\bar{S})+\bar{\theta} S_{t}\right\} \\
& \leq-\iint_{\Omega_{j} \cap \Omega_{t_{0}, t}}\left\{\bar{v}_{x}(p-\bar{p})-\bar{p}_{\tau} \bar{v}_{x}(\tau-\bar{\tau}) d x d s+\bar{\theta}_{\tau} \bar{v}_{x}(S-\bar{S})\right\} \\
\leq & -\iint_{\Omega_{j} \cap \Omega_{t_{0}, t}} \bar{v}_{x}\left(p-\bar{p}-\bar{p}_{\tau}\left(\tau_{a . c .}-\bar{\tau}\right)-\bar{p}_{S}(S-\bar{S})\right) d x d s+\iint_{\Omega_{j} \cap \Omega_{t_{0}, t}} \bar{v}_{x} \bar{p}_{\tau} \tau_{\text {sing }} \\
\leq & 0,
\end{aligned}
$$

since $\bar{p}_{\tau}<0$ and $\bar{v}_{x}$ is bounded and $\bar{v}_{x}(t, x) \geq 0$ everywhere over $\Omega_{1}$ and $\Omega_{3}$, and $\nabla_{W^{p}}^{2} p>0$. 
Step 4. Putting all these estimates together, we have

$$
\begin{aligned}
& \int_{|x| \leq X}\left(|v(t, x)-\bar{v}(t, x)|^{2}+\left|W_{a . c .}(t, x)-\bar{W}(t, x)\right|^{2} \omega(t, x)\right) d x \\
& \quad \leq 2 \int_{\substack{|x| \leq X+K_{0}\left(t-t_{0}\right) \\
\sigma=t_{0}}}\left\{\eta(W)-\eta(\bar{W})-\bar{v}(v-\bar{v})+\bar{p}\left(\mu_{t_{0}}-\bar{\tau}\right)-\bar{\theta}(S-\bar{S})\right\} .
\end{aligned}
$$

Now, applying Corollary 4.1, we finally get (4.10).

Corollary 4.2. Let $W(t, x)$ and $\bar{W}(t, x)$ satisfy the conditions of Theorem 4.2 and $W_{0}(x)=\bar{W}_{0}(x)$. Then $\tau(t, x)$ is absolutely continuous with respect to $\mathcal{L}^{2}$ in $\Pi_{T}$ and $W(t, x)=\bar{W}(t, x)$ a.e. in $\Pi_{T}$.

Proof. From Theorem 4.2, we have

$$
W_{a . c .}(t, x)=\bar{W}(t, x), \quad \text { a.e. in } \Pi_{T} .
$$

Hence, $\tau_{\text {sing }}$ must satisfy the weak formulation of

$$
\tau_{t}=0,\left.\quad \tau\right|_{t=0}=0,
$$

for the test functions in $C_{0}^{1}\left(\Pi_{T}^{*}\right)$. In particular, there exists $y \in B V_{l o c}\left(\Pi_{T}\right)$ such that

Therefore,

$$
\partial_{x} y=\tau_{\text {sing }}, \quad \partial_{t} y=0 .
$$

$$
\tau_{\text {sing }}=d t \otimes \nu_{t}(x)
$$

where $\nu_{t}(\cdot)=\frac{d y}{d x}(t, \cdot)$, a.e. $t \in(0, T)$. Since $y$ does not depend on $t$, we have that $\nu_{t}$ also does not depend on $t$, say, $\nu_{t}(x)=\nu_{0}(x)$ and

$$
\tau_{\text {sing }}=d t \otimes \nu_{0}
$$

Furthermore, since $\nu_{t} \rightarrow 0$ as $t \rightarrow 0$, we conclude

$$
\nu_{0} \equiv 0
$$

We now consider the case that the Riemann solution, with the initial condition (4.8), has a vacuum line at $x=0$. In this case, the Riemann solution $\bar{W}(t, x)$ has the following form:

$$
\bar{W}(x, t)= \begin{cases}W_{L}, & x / t<\xi_{L}, \\ R_{1}(x / t), & \xi_{L} \leq x / t<0, \\ \left(\left(\bar{v}_{1}+\bar{v}_{2}\right) / 2,\left(\bar{v}_{2}-\bar{v}_{1}\right) t d t \otimes \delta_{0}(x),\left(\bar{S}_{L}+\bar{S}_{R}\right) / 2\right), & x=0, \\ R_{3}(x / t), & 0<x / t \leq \xi_{R}, \\ W_{R}, & x / t>\xi_{R} .\end{cases}
$$

Here $R_{1}(x / t)$ and $R_{3}(x / t)$ are as above the rarefaction waves of the first and third characteristic families, respectively, $\bar{v}_{1}=\lim _{\xi \rightarrow 0_{-}} \bar{v}(\xi), \bar{v}_{2}=\lim _{\xi \rightarrow 0_{+}} \bar{v}(\xi)$, and $\delta_{0}(x)$ is the Dirac measure over $\mathbb{R}$ concentrated at 0 . It is easy to check that $\bar{W}(t, x)$ is a distributional solution of (1.1)-(1.3) and (4.4). The values of $\bar{v}$ and $\bar{S}$ on the line $x=0$ could be taken as any other constants instead of $\frac{\bar{v}_{1}+\bar{v}_{2}}{2}$ and $\frac{\bar{S}_{L}+\bar{S}_{R}}{2}$, 
respectively, while the formula of $\bar{\tau}$ at $x=0,\left(\bar{v}_{2}-\bar{v}_{1}\right) t d t \otimes \delta_{0}(x)$, is dictated by the fact that (1.1) must hold in the sense of distributions.

Theorem 4.3. Let $\bar{W}(t, x)$ be a Riemann solution containing vacuum as described in (4.12). Let $W(t, x)$ be a distributional entropy solution of (1.1)-(1.3) and (4.4) in $\Pi_{T}$ with $W_{0} \in L^{\infty}\left(\mathbb{R} ; \mathbb{R}^{3}\right)$. Then there exist positive constants $C$ and $K_{0}$, and a function $\omega \in L^{\infty}\left(\Pi_{T}\right)$, positive a.e. in $\Pi_{T}$, such that, for all $X>0$ and a.e. $t>0$,

$$
\begin{aligned}
\int_{|x| \leq X} & \left(|v(t, x)-\bar{v}(t, x)|^{2}+\left|W_{a . c .}^{p}(t, x)-\bar{W}^{p}(t, x)\right|^{2} \omega(t, x)\right) d x \\
& \leq C \int_{|x| \leq X+K_{0} t}\left(\left|v_{0}(x)-\bar{v}_{0}(x)\right|^{2}+\left|W_{0}^{p}(x)-\bar{W}_{0}^{p}(x)\right|^{2} \omega(0, x)\right) d x .
\end{aligned}
$$

Proof. Let $F_{1}, F_{2}$, and $F_{3}$ be as in the proof of Theorem 4.2. We observe that, since $\bar{p}=0$ for $x=0$, we have

$$
F_{2}=-\bar{p}\left(\tau-\bar{\tau}_{a . c .}, \bar{v}-v\right),
$$

so that the analysis for $F_{2}$ remains the same. Also, nothing needs to be changed concerning $F_{3}$. As for $F_{1}=\bar{v}(v-\bar{v}, p-\bar{p})$, we have a new aspect which is the fact that $\bar{v}(t, x)$ is discontinuous at $l$. Then we have

$$
\operatorname{div} F_{1}\left(l_{t_{0}, t}\right)=\left[\left\langle\left. F_{1} \cdot \nu\right|_{t_{t_{0}, t}}, 1\right\rangle\right]
$$

where $l_{t_{0}, t}=l \cap \Omega_{t_{0}, t}$ and again $\left.\nu\right|_{l}=(0,1)$. Let $p_{-}(t, x)$ and $p_{+}(t, x)$ denote the functions in $L^{\infty}(l)$, given by the theory of $\mathcal{D} \mathcal{M}^{\infty}$ fields developed in [5], such that

$$
\begin{aligned}
& \left\langle p_{-}, \zeta\right\rangle=\left\langle\left.(v, p) \cdot \nu\right|_{l}, \zeta\right\rangle_{l}, \\
& \left\langle p_{+}, \zeta\right\rangle=\left\langle\left.(v, p) \cdot(-\nu)\right|_{l}, \zeta\right\rangle_{l}, \quad \text { for any } \zeta \in C_{0}(l) .
\end{aligned}
$$

Hence, we have

$$
\operatorname{div} F_{1}\left(l_{t_{0}, t}\right)=v_{2} \int_{t_{0}}^{t} p_{+}(s) d s-v_{1} \int_{t_{0}}^{t} p_{-}(s) d s .
$$

On the other hand,

$$
p_{+}(s)=p_{-}(s), \quad \text { a.e. } s>0, \quad \text { and } \quad v_{2}>v_{1},
$$

where the first follows from (1.2) and the second is a consequence of the construction of the Riemann solution containing vacuum. Therefore, we conclude

$$
\operatorname{div} F_{1}\left(l_{t_{0}, t}\right) \geq 0 \text {. }
$$

The remaining of the proof follows exactly as in Theorem 4.2.

Again, we have the following corollary.

Corollary 4.3. Let $\bar{W}(t, x)$ and $W(t, x)$ satisfy the hypotheses of Theorem 4.3 and $W_{0}(x)=\bar{W}_{0}(x)$. Then $(v, S)(t, x)=(\bar{v}, \bar{S})(t, x), \mathcal{L}^{2}$-a.e. in $\Pi_{T}$, and $\tau=\bar{\tau}$ in $\mathcal{M}\left(\Pi_{T}\right)$.

Proof. From Theorem 4.3, we deduce that $W_{a . c}(t, x)=\bar{W}_{a . c}(t, x), \mathcal{L}^{2}$-a.e. in $\Pi_{T}$. Thus, as in the proof of Corollary 4.2, we deduce that $\tau_{\text {sing }}$ must be concentrated at $\{x=0\}$. Then $\tau_{\text {sing }}$ must be equal to $\left(\bar{v}_{2}-\bar{v}_{1}\right) t d t \otimes \delta_{0}(x)$ as a consequence of (1.1) in the sense of distributions. 


\section{Proof of Theorem 4.1}

In this section, we give a detailed proof of Theorem 4.1. The arguments are strongly motivated by those in Wagner [25].

Proof. We divide the proof into ten steps.

Step 1. There exists $y \in B V_{l o c}([0, \infty) \times \mathbb{R})$ such that

$$
\partial_{x} y=\tau, \quad \partial_{t} y=v
$$

in the sense of distributions for $t>0$.

Indeed, let $\omega^{\varepsilon}$ be a positive symmetric mollifier in $\mathbb{R}^{2}$, and set $\tau^{\varepsilon}=\tau * \omega^{\varepsilon}$ and $v^{\varepsilon}=v * \omega^{\varepsilon}$, where we have extended $\tau$ and $v$ as zero for $t<0$. Define

$$
y^{\varepsilon}(t, x)=\int_{0}^{x} \tau^{\varepsilon}(t, s) d s+\int_{0}^{t} v^{\varepsilon}(\sigma, 0) d \sigma .
$$

Then $y^{\varepsilon} \in B V \cap C^{1}\left(\mathbb{R}_{+}^{2}\right)$. We easily check that $y^{\varepsilon}(t, x)$ satisfies

$$
\partial_{x} y^{\varepsilon}=\tau^{\varepsilon}, \quad \partial_{t} y^{\varepsilon}=v^{\varepsilon}, \quad t>\varepsilon .
$$

Now, $\left\|y^{\varepsilon}\right\|_{B V(\Omega)} \leq M_{\Omega}$, for any open set $\Omega \Subset \mathbb{R}_{+}^{2}$, where $M_{\Omega}$ is a positive constant independent of $\varepsilon$. Hence, by the compact embedding of $B V(\Omega)$ into $L^{1}(\Omega)$, there exists $y \in B V_{l o c}\left(\mathbb{R}_{+}^{2}\right)$ such that

$$
y^{\varepsilon}(t, x) \rightarrow y(t, x), \quad \text { in } \quad L_{l o c}^{1}\left(\mathbb{R}_{+}^{2}\right),
$$

by passing to a subsequence if necessary. Clearly, $y(t, x)$ satisfies $(5.1)$ in the sense of distributions in $\mathbb{R}_{+}^{2}$.

Step 2. The measure $\tau(t, x)$ admits a slicing of the form $\tau=d t \otimes \mu_{t}$, where, for $\mathcal{L}^{1}$-a.e. $t>0, \mu_{t} \in \mathcal{M}^{+}(\mathbb{R})$.

Let $y(t, x)$ be a solution of (5.1). Since $y \in B V_{l o c}\left(\mathbb{R}_{+}^{2}\right)$, then, for a.e. $t>0$, $y(t, \cdot) \in B V_{l o c}(\mathbb{R})$. Hence,

$$
\tau=d t \otimes \frac{d y}{d x}(t, \cdot) .
$$

Step 3. The points $(t, x) \in \mathbb{R}_{+}^{2}$ such that $\mu_{t}(x)>0$, with the possible exception of a set of $\mathcal{H}^{1}$-measure zero, form a countable union of vertical line segments.

Again, since $y \in B V_{l o c}\left(\mathbb{R}_{+}^{2}\right)$, then, for a.e. $x \in \mathbb{R}, y(\cdot, x) \in B V_{l o c}\left(\mathbb{R}_{+}\right)$. Hence, $\partial_{t} y$ admits a slicing as

$$
\partial_{t} y=\frac{d y}{d t}(\cdot, x) \otimes d x=v(\cdot, x) d t \otimes d x .
$$

That is, for a.e. $x \in \mathbb{R}, y(\cdot, x)$ is a Lipschitz function on $[0, \infty)$ whose derivative is $v(\cdot, x)$. On the other hand, the jump set of $y(t, x)$, with the possible exception of a set of $\mathcal{H}^{1}$-measure zero, is a countable union of $C^{1}$ curves $\left\{l_{k}\right\}_{k \in \mathbb{N}}$, by the structure theory of $B V$ functions (see, e.g., $[12,13]$ ). We then conclude that the lines $l_{k}$ must be vertical, because, otherwise, we would have a subset $A \subset \mathbb{R}$ of positive measure such that, for $x \in A, \frac{d y}{d t}(\cdot, x)$ would be a singular measure, rather than an $L^{\infty}$ function $v(\cdot, x)$, which proves the assertion.

Observe that $\mu_{t} \rightarrow \tau_{0}$ as $t \rightarrow 0+$, which follows from standard arguments by choosing suitable test functions in (4.1).

Step 4. Let $x_{0} \in \mathbb{R}$ be such that $\tau_{0}\left(x_{0}\right)=\mu_{t}\left(x_{0}\right)=0$, a.e. $t>0$, and $x_{0}$ is a Lebesgue point of $\int_{0}^{t} v(\sigma, \cdot) d \sigma$, for all rational $t>0$ and hence all fixed $t>0$. 
Then there exists a solution of (5.1) satisfying

$$
\lim _{t \rightarrow 0+} y(t, x)=\int_{x_{0}}^{x} \tau_{0}, \quad \text { for a.e. } x \in \mathbb{R} .
$$

Consider a mollifier $\omega^{\varepsilon}$, with $\varepsilon=\left(\varepsilon_{1}, \varepsilon_{2}\right)$, of the form $\omega^{\varepsilon}(t, x)=\delta^{\varepsilon_{1}}(t) \delta^{\varepsilon_{2}}(x)$, where $\delta^{\varepsilon_{j}}, j=1,2$, are standard positive symmetric mollifiers in $\mathbb{R}$. If $y^{\varepsilon}(t, x)$ is defined by (5.2), with 0 replaced by $x_{0}$, then sending $\varepsilon_{1}$ to 0 first and next letting $\varepsilon_{2} \rightarrow 0$ yield

$$
\lim _{\varepsilon_{2} \rightarrow 0} \lim _{\varepsilon_{1} \rightarrow 0} y^{\varepsilon}(t, x)=\int_{x_{0}}^{x} \mu_{t}+\int_{0}^{t} v\left(\sigma, x_{0}\right) d \sigma, \quad \text { a.e. }(t, x) \in \mathbb{R}_{+}^{2} .
$$

Hence,

$$
y(t, x)=\int_{x_{0}}^{x} \mu_{t}+\int_{0}^{t} v\left(\sigma, x_{0}\right) d \sigma,
$$

provides the desired solution because of Step 4 .

Now, since, for each $t \geq 0, y(t, x)$ is a strictly increasing function of $x$, there exists a well-defined monotone increasing continuous function $x(t, y)$ such that

$$
x(t, y(t, x))=x, \quad(t, x) \in \mathbb{R}_{+}^{2},
$$

where we have defined $y(t, x)$ on the vacuum lines by, say, $(y(t, x+0)+y(t, x-0)) / 2$.

Let $Q$ and $T$ be the transformations of $\mathbb{R}_{+}^{2}, Q(t, x)=(t, y(t, x))$ and $T(t, y)=$ $(t, x(t, y))$ so that $T(Q(t, x))=(t, x)$. Observe that, given an open rectangle $R=$ $\left(t_{1}, t_{2}\right) \times(a, b) \subset \mathbb{R}_{+}^{2}$, if $a$ and $b$ are such that $y(t, a)$ and $y(t, b)$ are continuous functions of $t$, then $Q(R)$ is an open set in $\mathbb{R}_{+}^{2}$, which implies that $T$ is continuous in $\mathbb{R}_{+}^{2}$. Let

$$
T_{0}(x)=\lim _{t \rightarrow 0+} y(t, x), \quad Q_{0}(y)=\lim _{t \rightarrow 0_{+}} x(t, y) .
$$

Step 5. $T_{\#} \mathcal{L}^{2}=\tau$ and $T_{0 \#} \mathcal{L}^{1}=\tau_{0}$, with notation from [13].

In fact, for a.e. $t>0, y(t, x)$ is a strictly increasing $B V_{l o c}$ function of the variable $x$, and $\tau=d t \otimes \mu_{t}(x)$ with $\mu_{t}(x)=\frac{d y}{d x}(t, \cdot)$. Now, $T(t, y)=(t, x(t, y))$ and, for any open interval $(a, b)$ and for each fixed $t>0$,

$$
x(t, \cdot)^{-1}(a, b)=(y(t, a+0), y(t, b-0))=\tau(a, b) .
$$

Hence, the measure $T_{\#} \mathcal{L}^{2}$ equals $d t \otimes \tilde{\mu}_{t}$, where $\tilde{\mu}_{t}$ is the Stieltjes measure associated with $y(t, x)$, that is, $\tilde{\mu}_{t}=\mu_{t}$, which implies $T_{\#} \mathcal{L}^{2}=\tau$. Similarly, we obtain $T_{0 \#} \mathcal{L}^{1}=\tau_{0}$.

Step 6. The map $T$ is proper and onto.

The fact that $T$ is onto follows from the fact that it is the inverse of $Q$ which is defined everywhere in $\mathbb{R}_{+}^{2}$. Now, since $Q(t, x)$ is a continuous function of $t$, for a.e. $x \in \mathbb{R}$, given any compact $K \subset \mathbb{R}_{+}^{2}$, we may find a rectangle $\left[t_{1}, t_{2}\right] \times[a, b]$ containing $K$ such that $Q(t, a)$ and $Q(t, b)$ are continuous functions of $t$. Hence, the set

$$
\left\{(t, y) \mid y(t, a) \leq y \leq y(t, b), t_{1} \leq t \leq t_{2}\right\}
$$

is compact and contains $T^{-1}(K)$, which is then also compact by the continuity of $T$.

Let $\rho=Q_{\#} \mathcal{L}^{2}$. Let $\tilde{\rho}$ be the density of $\mathcal{L}^{2}$ with respect to $\tau$ in $\mathbb{R}_{+}^{2}$ and let $\tilde{\tau}$ be the density of $\tau_{\text {a.c. }}$. with respect to $\mathcal{L}^{2}$ so that $\tilde{\rho} \tilde{\tau}=1, \mathcal{L}^{2}$-a.e. in $\mathbb{R}_{+}^{2}$. The following result was proved by Wagner ([25], p.132).

Step \%. $\rho=\partial_{y} x=\tilde{\rho} \circ T$. 
Indeed, $\rho=Q_{\#} \mathcal{L}^{2}$ admits a slicing of the form $\rho=d t \otimes \tilde{\mu}_{t}$, where $\tilde{\mu}_{t}$ is the Stieltjes measure associated with the inverse of $y(t, \cdot)$, which is $x(t, y)$, and hence $\tilde{\mu}_{t}=\partial_{y} x$. Furthermore, The Lipschitz continuity of $x(t, y)$ yields

$$
\rho=\partial_{y} x
$$

where as usual we have identified absolutely continuous measures with their densities, with respect to the corresponding Lebesgue measure. Also, we have

$$
T_{\#} \rho=T_{\#} Q_{\#} \mathcal{L}^{2}=\mathcal{L}^{2}=\tilde{\rho} \tilde{\tau}=\tilde{\rho} \tau=\tilde{\rho} T_{\#} \mathcal{L}^{2} .
$$

Now, for all $\phi \in C_{0}((0, \infty) \times \mathbb{R})$,

$$
\langle\rho, \phi\rangle=\left\langle T_{\#} \rho, \phi \circ Q\right\rangle
$$

while

$$
\langle\tilde{\rho} \circ T, \phi\rangle=\left\langle\tilde{\rho} T_{\#} \mathcal{L}^{2}, \phi \circ Q\right\rangle,
$$

which implies

$$
\rho=\tilde{\rho} \circ T,
$$

where we have used that $\rho=0$ a.e. over $T^{-1}(V)$ and $V$ is the union of the vacuum lines, because of the first part of the assertion.

Now, let $\rho_{0}=Q_{0 \#} \mathcal{L}^{1}$ and $\underline{v}(t, y)=v(T(t, y))$.

Step 8. For all $\phi \in C_{0}^{1}\left(\mathbb{R}^{2}\right)$, we have

$$
\iint_{t>0}\left(\rho \phi_{t}+\rho \underline{v} \phi_{y}\right) d t d y+\int_{t=0} \phi(0, y) \rho_{0}(y) d y=0 .
$$

Observe that $\rho$ and $\rho_{0}$ are uniformly bounded since $\rho=\tilde{\rho} \circ T, \tilde{\rho} \tilde{\tau}=1$, and $\tilde{\tau} \geq c$ for some $c>0$. The same holds for $\rho_{0}$. Now, for any $\phi \in C_{0}^{1}\left(\mathbb{R}^{2}\right)$, we have

$$
\begin{aligned}
& \iint_{t>0}\left(\phi_{t}+\phi_{y} \underline{v}\right) \rho d t d y+\int_{t=0} \phi(0, y) \rho_{0}(y) d y \\
& =\iint_{t>0}\left(\phi_{t} \circ Q+\phi_{y} \circ Q \underline{v} \circ Q\right) d t d x+\int_{t=0} \phi\left(0, Q_{0}(x)\right) d x \\
& =\iint_{t>0}\left(\phi_{t} \circ Q+v \phi_{y} \circ Q\right) d t d x+\int_{t=0} \phi\left(0, Q_{0}(x)\right) d x \\
& =\iint_{t>0} \partial_{t}(\phi \circ Q) d t d x+\int_{t=0} \phi\left(0, Q_{0}(x)\right) d x=0
\end{aligned}
$$

where we have used that $\underline{v} \circ Q=v$. In particular, we have

$$
\rho_{t}+(\rho \underline{v})_{y}=0
$$

in the sense of distributions. Now, let $x_{0} \in \mathbb{R}$ be such that $y\left(t, x_{0}\right)$ is a Lipschitz continuous function on $[0, \infty)$ and, for a.e. $t, y\left(t, x_{0}\right)$ is a Lebesgue point for both $\rho(t, y)$ and $\underline{v}(t, y)$.

Step 9. There exists a solution $\bar{x}(t, y)$ of

$$
\partial_{y} x=\rho, \quad \partial_{t} x=-\rho \underline{v},
$$

such that $\bar{x}\left(t, y\left(t, x_{0}\right)\right)=x_{0}$. In particular, $\bar{x}(t, y)=x(t, y)$.

Indeed, let $\rho^{\varepsilon}=\rho * \omega^{\varepsilon}$ and $(\rho \underline{v})^{\varepsilon}=(\rho \underline{v}) * \omega^{*}$, where $\omega^{\varepsilon}$ is a standard mollifier. Set

$$
x^{\varepsilon}(t, y)=\int_{y\left(t, x_{0}\right)}^{y} \rho^{\varepsilon}(t, s) d s+x_{0}
$$


Then $x^{\varepsilon}(t, y)$ is a Lipschitz function satisfying

$$
\begin{aligned}
& \partial_{y} x^{\varepsilon}=\rho^{\varepsilon}(t, y), \\
& \partial_{t} x^{\varepsilon}=-(\rho \underline{v})^{\varepsilon}(t, y)-\rho^{\varepsilon}\left(t, y\left(t, x_{0}\right)\right) \underline{v}\left(t, y\left(t, x_{0}\right)\right)+(\rho \underline{v})^{\varepsilon}\left(t, y\left(t, x_{0}\right)\right) .
\end{aligned}
$$

Also,

$$
\left|x^{\varepsilon}(t, y)-x_{0}\right| \leq M\left|y-y\left(t, x_{0}\right)\right| .
$$

Hence, $x^{\varepsilon}(t, y)$ converges in $L_{l o c}^{1}\left(\mathbb{R}_{+}^{2}\right)$ to a Lipschitz function $\bar{x}(t, y)$ which satisfies

$$
\left|\bar{x}(t, y)-x_{0}\right| \leq M\left|y-y\left(t, x_{0}\right)\right| \text {. }
$$

Thus, we have $\bar{x}\left(t, y\left(t, x_{0}\right)\right)=x_{0}$. Since

$$
\left|\rho^{\varepsilon}\left(t, y\left(t, x_{0}\right)\right) \underline{v}\left(t, y\left(t, x_{0}\right)\right)-(\rho \underline{v})^{\varepsilon}\left(t, y\left(t, x_{0}\right)\right)\right| \rightarrow 0, \quad \text { as } \varepsilon \rightarrow 0,
$$

for a.e. $t>0$, we conclude that $\bar{x}(t, y)$ satisfies (5.7). Now, since $\partial_{y} x(t, y)=\rho(t, y)$ and $x\left(t, y\left(t, x_{0}\right)\right)=x_{0}$, we must have $x(t, y)=\bar{x}(t, y)$.

Step 10. Equation (4.1) holds for any $\tau$-test function $\phi(t, x)$.

Indeed, for any $\tau$-test function $\phi(t, x)$, we have

$$
\begin{aligned}
& \iint_{t>0}\left(\phi_{t} \tau-v \phi_{x} d t d x\right)+\int_{t=0} \phi(0, x) \tau_{0} \\
& =\iint_{t>0}\left(\phi_{t}-v \tilde{\rho} \phi_{x}\right) \tau+\int_{t=0} \phi(0, x) \tau_{0} \\
& =\iint_{t>0}\left(\phi_{t} \circ T-\phi_{x} \circ T \rho \underline{v}\right) d t d y+\int_{t=0} \phi \circ T_{0} d y \\
& =\iint_{t>0} \frac{\partial}{\partial t}(\phi \circ T) d t d y+\int_{t=0} \phi \circ T_{0} d y \\
& =-\int_{\mathbb{R}} \lim _{\delta \rightarrow 0} \phi \circ T(y, \delta) d y+\int_{t=0} \phi \circ T_{0} d y=0 .
\end{aligned}
$$

Acknowledgments. Gui-Qiang Chen's research was supported in part by the National Science Foundation under Grants DMS-0204225, INT-9987378, and INT9726215. Hermano Frid's research was supported in part by $\mathrm{CNPq}$ through the grants 352871/96-2, 465714/00-5, 479416/01-0 and FAPERJ through the grant E$26 / 151.890 / 2000$.

\section{REFERENCES}

[1] Anzellotti, G., Pairings between measures and functions and compensated compactness, Ann. Mat. Pura Appl. 135 (1983), 293-318.

[2] Baiocchi, C. and Capelo, A., Variational and Quasi-Variational Inequalities, Applications to Free-Boundary Problems, Vols. 1,2, Wiley: Chichester-New York, 1984.

[3] Bianchini, S. and Bressan, A., Vanishing viscosity solutions of nonlinear hyperbolic systems, Preprint, SISSA, Italy, 2001.

[4] Brezzi, F. and Fortin, M., Mixed and Hydrid Finite Element Methods, Springer-Verlag: New York, 1991.

[5] Chen, G.-Q. and Frid, H., Divergence-measure fields and conservation laws, Arch. Rational Mech. Anal. 147 (1999), 89-118.

[6] Chen, G.-Q., and Frid, H., Decay of entropy solutions of nonlinear conservation laws, Arch. Rat. Mech. Anal. 146 (1999), 95-127.

[7] Chen, G.-Q. and Frid, H., Large-time behavior of entropy solutions of conservation laws, J. Diff. Eqs. 152 (1999), 308-357. 
[8] Chen, G.-Q. and Frid, H., Uniqueness and asymptotic stability of Riemann solutions for the compressible Euler equations, Trans. Amer. Math. Soc. 353 (2001),1103-1117.

[9] Chen, G.-Q., Frid, H., and Y. Li, Uniqueness and stability of Riemann solutions with large oscillation in gas dynamics, Commun. Math. Phys. 2002 (to appear).

[10] Dafermos, C. M., Hyperbolic Conservation Laws in Continuum Physics, Springer-Verlag: Berlin, 1999.

[11] DiPerna, R., Uniqueness of solutions to hyperbolic conservation laws, Indiana Univ. Math. J. 28 (1979), 137-188.

[12] Evans, L. C. and Gariepy, R. F., Lecture Notes on Measure Theory and Fine Properties of Functions, CRC Press: Boca Raton, Florida, 1992.

[13] Federer, H., Geometric Measure Theory, Springer-Verlag: Berlin-Heidelberg-New York, 1969.

[14] Gagliardo, E., Caratterizioni delle tracce sulla frontiera relativa ad alcune classi di funzioni in $n$ variabli, Rend. Sem. Mat. Univ. Padova 27 (1957), 284-305.

[15] Glimm, J., Solutions in the large for nonlinear hyperbolic systems of equations, Comm. Pure Appl. Math. 18 (1965), 95-105.

[16] Lax, P. D., Hyperbolic Systems of Conservation Laws and the Mathematical Theory of Shock Waves, CBMS. 11, SIAM, Philadelphia, 1973.

[17] Lax, P. D., Shock waves and entropy, In: Contributions to Functional Analysis, ed. E. A. Zarantonello, Academic Press, New York, 1971, pp. 603-634.

[18] Liu, T.-P. and Smoller, J., On the vacuum state for the isentropic gas dynamics equations, Adv. Appl. Math. 1 (1980), 345-359.

[19] Perthame, B. Kinetic Formulation of Conservation Laws, Lecture Notes, Ecole Normale Supérieure, Paris, 2001.

[20] Rodrigues, J.-F., Obstacle Problems in Mathematical Physics, North-Holland Mathematics Studies 134, Elsevier Science Publishers B.V. 1987.

[21] Schwartz, L., Théorie des Distributions (2 volumes), Actualites Scientifiques et Industrielles 1091, 1122, Herman: Paris, 1950-51.

[22] Serre, D., Systems of Conservation Laws, Vols. 1,2, Cambridge University Press: Cambridge, 1999, 2000.

[23] Stein, E., Singular Integrals and Differentiability Properties of Functions, Princeton Univ. Press: Princeton, NJ, 1970.

[24] Volpert, A. I., The space BV and quasilinear equations, Mat. Sb. (N.S.) 73 (1967), 255-302, Math. USSR Sbornik 2 (1967), 225-267 (in English).

[25] Wagner, D., Equivalence of the Euler and Lagrange equations of gas dynamics for weak solutions, J. Diff. Eqs. 68 (1987), 118-136.

[26] Whitney, H., Geometric Integration Theory, Princeton Univ. Press: Princeton, NJ, 1957.

[27] Whitney, H., Analytic extensions of differentiable functions defined in closed sets, Trans. Amer. Math. Soc. 36 (1934), 63-89.

[28] Ziemer, W. P., Cauchy flux and sets of finite perimeter, Arch. Rational Mech. Anal. 84 (1983), 189-201.

[29] Ziemer, W. P., Weakly Differentiable Functions: Sobolev Spaces and Functions of Bounded Variation, Springer-Verlag: New York, 1989.

(G.-Q. Chen) Department of Mathematics, Northwestern University, 2033 Sheridan Road, Evanston, Illinois 60208-2730, USA

E-mail address: gqchen@math.northwestern.edu

(H. Frid) Instituto de Matemática Pura e Aplicada - impa, Estrada Dona Castorina, 110, Rio de Janeiro, RJ 22460-320, BraziL

E-mail address: hermano@impa.br 